\title{
Relations between graphs
}

\author{
Jan Hubička
}

Computer Science Institute of Charles University, Univerzita Karlova v Praze

Malostranské nám. 25, 11800 Praha 1, Czech Republic

\section{Jürgen Jost}

Max Planck Institute for Mathematics in the Sciences Inselstrasse 22, D-04103 Leipzig, Germany

Department of Mathematics, University of Leipzig, D-04081 Leipzig, Germany

Santa Fe Institute, 1399 Hyde Park Rd., Santa Fe, NM 87501, USA

\section{Yangjing Long}

Max Planck Institute for Mathematics in the Sciences Inselstrasse 22, D-04103 Leipzig, Germany

\section{Peter F. Stadler}

Bioinformatics Group, Department of Computer Science, and Interdisciplinary Center for Bioinformatics, University of Leipzig, Härtelstraße 16-18, D-04107 Leipzig, Germany

Max Planck Institute for Mathematics in the Sciences Inselstrasse 22, D-04103 Leipzig, Germany

Santa Fe Institute, 1399 Hyde Park Rd., Santa Fe, NM 87501, USA

Fraunhofer Institut für Zelltherapie und Immunologie - IZI Perlickstraße 1, D-04103 Leipzig, Germany

Department of Theoretical Chemistry, University of Vienna

Währingerstraße 17, A-1090 Wien, Austria

Center for non-coding RNA in Technology and Health University of Copenhagen, Grønnegårdsvej 3, DK-1870 Frederiksberg C, Denmark

\section{Ling Yang}

School of Mathematical Sciences, Fudan University

No. 220 Handan Rd., 200433, Shanghai, China

Received 18 May 2012, accepted 24 October 2012, published online 14 January 2013

E-mail addresses: Jan.Hubicka@mff.cuni.cz (Jan Hubička), jost@mis.mpg.de (Jürgen Jost), ylong@mis.mpg.de (Yangjing Long), studla@bioinf.uni-leipzig.de (Peter F. Stadler), yanglingfd@fudan.edu.cn (Ling Yang) 


\begin{abstract}
Given two graphs $G=\left(V_{G}, E_{G}\right)$ and $H=\left(V_{H}, E_{H}\right)$, we ask under which conditions there is a relation $R \subseteq V_{G} \times V_{H}$ that generates the edges of $H$ given the structure of the graph $G$. This construction can be seen as a form of multihomomorphism. It generalizes surjective homomorphisms of graphs and naturally leads to notions of R-retractions, Rcores, and R-cocores of graphs. Both R-cores and R-cocores of graphs are unique up to isomorphism and can be computed in polynomial time.
\end{abstract}

Keywords: Generalized surjective graph homomorphism, R-reduced graph, R-retraction, binary relation, multihomomorphism, $R$-core, cocore.

Math. Subj. Class.: 05C60, 05C76

\title{
1 Introduction
}

\subsection{Motivation}

Graphs are frequently employed to model natural or artificial systems [3, 11]. In many applications separate graph models have been constructed for distinct, but at least conceptually related systems. One might think, e.g., of traffic networks for different means of transportation (air, ship, road, railroad, bus). In the life sciences, elaborate network models are considered for gene expression and the metabolic pathways regulated by these genes, or for the co-occurrence of protein domains within proteins and the physical interactions of proteins among each other.

Let us consider an example. Most proteins contain several functional domains, that is, parts with well-characterized sequence and structure features that can be understood as functional units. Protein domains for instance mediate the catalytic activity of an enzyme and they are responsible for specific binding to small molecules, nucleic acids, or other proteins. Databases such as SuperFamily compile the domain composition of a large number of proteins. We can think of these data as a relation $R \subset D \times P$ between the set $D$ of domains and the set of $P$ proteins which contain them. Protein-protein interaction networks (PPIs) have been empirically determined for several species and are among the best-studied biological networks [16]. From this graph, which has $P$ as its vertex set, and the relation $R$ we can obtain a new graph whose vertex set are the protein domains $D$, with edges connecting domains that are found in physically interacting proteins. This "domain interaction graph" conveys information e.g. on the functional versatility of protein complexes. On the other hand, we can use $R$ to construct the domain-cooccurrence networks (DCNs) [14] as simple relational composition $R \circ R^{+}$. In examples like these, the detailed connections between the various graphs have remained unexplored. In fact, there may not be a meaningful connection between some of them, e.g. between PPIs and DCNs, while in other cases there is a close connection: the domain interaction graph, for example, is determined by the PPI and $R$.

A second setting in which graph structures are clearly related to each other is coarsegraining. Here, sets of vertices are connected to a single coarse-grained vertex, with coarsegrained edges inherited from the original graph. In the simplest case, we deal with quotient graphs [15], although other, less stringent constructions are conceivable. Similarly, we would expect that networks that are related by some evolutionary process retain some sort 


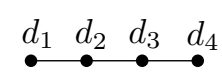

Graph $G$

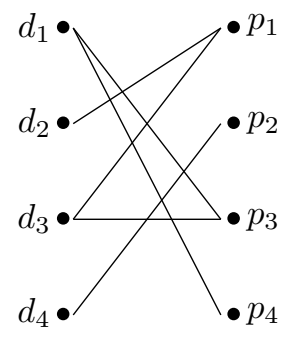

Relation $R$

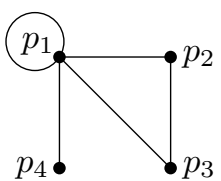

Graph $G * R$

Figure 1: The graph $G * R$ is determined by the graph $G$ and the relation $R$.

of structural relationship.

\subsection{Main definitions}

A well-defined mathematical problem is hidden in this setting: Given two networks, can we identify whether they are related in meaningful ways? The usual mathematical approach to this question, namely to ask for the existence of structure-preserving maps, appears to be much too restrictive. Instead, we set out here to ask if there is a relation between the two networks that preserves structures in a less restrained sense.

The idea is to transfer edges from a graph $G$ to a graph $H$ with the help of a relation $R$ between the vertex set $V$ of $G$ and the vertex set $B$ of $H$. In this context, $R$ is simply a set of pairs $(v, b)$, with $v \in V, b \in B$. Since graphs can be regarded as representations of binary relations, we can also consider $G$ as a relation on its vertex set, with $(x, y) \in G$ if and only if $x$ and $y$ are connected by an edge of $G$. We then have the composition $G \circ R$ given by all pairs $(x, b)$ for which there exists a vertex $y \in V$ connected by an edge of $G$ to $x$ and $(y, b) \in R$. This, however, like $R$ is a relation between elements of different sets. In order to equip the target set $B$ with a graph structure, we simply connect elements $u$ and $v$ in $B$ if they stand in relation to connected elements of $G$. In the following, we give a formal definition, and we shall then relate it to the composition of relations just described.

A directed graph $G$ is a pair $G=\left(V_{G}, E_{G}\right)$ such that $E_{G}$ is a subset of $V_{G} \times V_{G}$. We denote by $V_{G}$ the set of vertices of $G$ and by $E_{G}$ the set of edges of $G$. We consider only finite graphs and allow loops on vertices.

An undirected graph (or simply a graph) $G$ is any directed graph such that $(u, v) \in E_{G}$ if and only if $(v, u) \in E_{G}$. We thus consider undirected graphs to be special case of directed graphs and we still allow loops on vertices. A simple graph is an undirected graph without loops.

Definition 1.1. Let $G=\left(V_{G}, E_{G}\right)$ be a graph, $B$ a finite set, and $R \subseteq V \times B$ a binary relation, where for every element $b \in B$, we can find an element $v \in V_{G}$ such that $(v, b) \in$ $R$. Then the graph $G * R$ has vertex set $B$ and edge set

$$
E_{G * R}=\left\{(u, v) \in B \times B \mid \text { there is }(x, y) \in E_{G} \text { and }(x, u),(y, v) \in R\right\} .
$$

An example of the $*$ operation is depicted in Fig. 1 .

Graphs with loops are not always a natural model, however, so that it may appear more appealing to consider the slightly modified definition. 
Definition 1.2. Let $G=\left(V_{G}, E_{G}\right)$ be a simple graph, $B$ a finite set, $R$ a binary relation, where for every element $b \in B$, we can find an element $v \in V_{G}$ such that $(v, b) \in R$. Then the (simple) graph $G \star R$ has vertex set $B$ and edge set

$$
E_{G \star R}=\left\{(u, v) \in B \times B \mid u \neq v \text { and there is }(x, y) \in E_{G} \text { and }(x, u),(y, v) \in R\right\} .
$$

We shall remark that these definitions remain meaningful for directed graphs, weighted graphs (where the weight of edge is a sum of weights of its pre-images) as well as relational structures. For simplicity, we restrict ourselves to undirected graphs (with loops). Most of the results can be directly generalized.

Graphs can be regarded as representations of symmetric binary relations. Using the same symbol for the graph and the relation it represents, we may re-interpret definition 1.1 as a conjugation of relations. $R^{+}$is the transpose of $R$, i.e., $(u, x) \in R^{+}$if and only if $(x, u) \in R$. The double composition $R^{+} \circ G \circ R$ contains the pair $(u, v)$ in $B \times B$ if and only if there are $x$ and $y$ such that $(u, x) \in R^{+},(y, v) \in R$, and $(x, y) \in E_{G}$. Thus

$$
G * R=R^{+} \circ G \circ R \text {. }
$$

Simple graphs, analogously, correspond to the irreflexive symmetric relations. For any relation $R$, let $R^{\iota}$ denote its irreflexive part, also known as the reflexive reduction of $R$. Since definition 1.2 explicitly excludes the diagonals, it can be written in the form

$$
G \star R=\left(R^{+} \circ G \circ R\right)^{\iota}
$$

We have $G \star R=(G * R)^{\iota}$, and hence $E_{G \star R} \subseteq E_{G * R}$. The composition $G * R$ is of particular interest when $G$ is also a simple graph, i.e., $G=G^{\iota}$.

The main part of this contribution will be concerned with the solutions of the equation $G * R=H$. The weak version, $G \star R=H$, will turn out to have much less convenient properties, and will be discussed only briefly in section 7 .

Throughout this paper we use the following standard notations and terms.

For relation $R \subseteq X \times Y$ we define by $R(x)=\{p \in Y \mid(x, p) \in R\}$ the image of $x$ under $R$ and $R^{-1}(p)=\{x \in X \mid(x, p) \in R\}$ the pre-image of $p$ under $R$.

The domain of $R$ is defined by $\operatorname{dom} R=\{x \in X \mid \exists p \in Y$ s.t. $(x, p) \in R\}$, and the image of $R$ is defined by $\operatorname{img} R=\{p \in Y \mid \exists x \in X$ s.t. $(x, p) \in R\}$. We say that the domain of $R$ is full if for any $x \in X$ we have $R(x) \neq \emptyset$. Analogously, the image is full if for any $p \in Y$ we have $R^{-1}(p) \neq \emptyset$.

Let $R \subseteq X \times Y$ is a binary relation, then $R$ is injective, if for all $x$ and $z$ in $X$ and $y$ in $Y$ it holds that if $(x, y) \in R$ and $(z, y) \in R$ then $x=z$. $R$ is functional, if for all $x$ in $X$, and $y$ and $z$ in $Y$ it holds that if $(x, y) \in R$ and $(x, z) \in R$ then $y=z$.

We denote by $I_{G}$ the identity map on $G$, i.e., $\left\{(x, x) \mid x \in V_{G}\right\}$.

Let $G=\left(V_{G}, E_{G}\right)$ be a graph and let $W \subseteq V_{G}$. The induced subgraph $G[W]$ has vertex set $W$ and $(x, y)$ is an edge of $G[W]$ if $x, y \in W$ and $(x, y) \in E_{G}$.

A graph $P_{k}$ is a path of length $k$. Similarly, $C_{k}$ is an (elementary) cycle of length $k$ with vertex set $\{0,1, \ldots, k-1\}$. Finally, $K_{k}$ is the complete (loopless) graph with $k$ vertices.

An isolated vertex is a vertex with degree 0 . Note that the vertex with a loop is not isolated in this sense. 


\subsection{Matrix multiplication}

The operation $*$ can also be formulated in terms of matrix multiplication. To see this, consider the following variant of the operation on weighted graphs.

Definition 1.3. If $G$ is a weighted graph, we use $w(x, y)$ to denote the weight between $x$ and $y$. Given a finite set $B$ and a binary relation $R \subseteq V_{G} \times B, G \circledast R$ is defined as a weighted graph $H$ with vertex set $B$, for any $u, v \in B, w(u, v)=\sum_{(x, u) \in R,(y, v) \in R} w(x, y)$.

Ignoring the weights, operations $*$ and $\circledast$ are equivalent.

Using the language of matrices, $G \circledast R=H$ can be interpreted as matrix multiplication:

$$
\mathbf{W}_{G \circledast R}=\mathbf{R}^{+} \mathbf{W}_{G} \mathbf{R}
$$

where $\mathbf{R}$ is the matrix representation of the relation $R$, i.e., $\mathbf{R}_{x u}=1$ if and only if $(x, u) \in$ $R$, otherwise $\mathbf{R}_{x u}=0, \mathbf{R}^{+}$denotes the transpose of $\mathbf{R}$, and $\mathbf{W}_{G}$ is the matrix of edge weights of $G$.

\subsection{Graph homomorphisms and multihomomorphisms}

The notion of relations between graphs is in many ways similar (but not equivalent) to the well studied notion of graph homomorphisms. The majority of our results focus on similarities and differences between those two concepts. We give here only the basic definitions of graph homomorphisms. For more details see [7].

A homomorphism from a graph $G$ to a graph $H$ is a mapping $f: V_{G} \rightarrow V_{H}$ such that for every edge $(x, y)$ of $G,(f(x), f(y))$ is an edge of $H$. Note that homomorphisms require loops in $H$ whenever $(x, y) \in E_{G}$ and $f(x)=f(y)$. In contrast, $f$ is a weak homomorphism if $(x, y) \in E_{G}$ implies that either $f(x)=f(y)$ or $(f(x), f(y)) \in E_{H}$. Every homomorphism from $G$ to $H$ induces also a weak homomorphism, but not conversely [9].

Since every homomorphism preserves adjacency, it naturally defines a mapping $f^{1}$ : $E_{G} \rightarrow E_{H}$ by setting $f^{1}((x, y))=(f(x), f(y))$ for all $(x, y) \in E_{G}$. If $f$ is surjective, we call $f$ a vertex surjective homomorphism, and if $f^{1}$ is surjective, we call $f$ an edge surjective homomorphism. $f$ is surjective homomorphism if it is both vertex- and edge-surjective [7].

A map $f: V_{G} \rightarrow V_{H}$ is, of course, a special case of a relation. This is seen by setting $F=\left\{(x, f(x)) \mid x \in V_{G}\right\}$. Hence, there is a surjective homomorphism from $G$ to $H$ if and only if there is a functional relation $F$ such that $G * F=H$. Another important connection to the graph homomorphisms is the following simple lemma.

Lemma 1.4. If $G * R=H$, and the domain of $R$ is full, then there is a homomorphism $f$ from $G$ to $H$ contained in $R$.

Proof. If $G * R=H$, then take any functional relation $f \subseteq R$, we have $G * f \subseteq H$, where $f$ is a homomorphism from $G$ to $H$.

Analogously, there is a weak surjective homomorphism from $G$ to $H$ if and only if there is a functional relation $F$ such that $G \star F=H$, and there is a weak homomorphism from $G$ to $H$ if there is a functional relation $F \subseteq V_{G} \times V_{H}$ such that $G \star F$ is a subgraph of $H$. The existence of relations between graphs thus can be seen as a proper generalization of graph homomorphisms or weak graph homomorphisms, respectively. 
Finally, a full homomorphism from a graph $G$ to a graph $H$ is a vertex mapping $f$ such that for distinct vertices $u$ and $v$ of $G$, we have $(u, v)$ an edge of $G$ if and only if $(f(u), f(v))$ is an edge of $H$, see [4].

Relation between graphs can be regarded also as a variant of multihomomorphisms. Multihomomorphisms are building blocks of Hom-complexes, introduced by Lovász, and are related to recent exciting developments in topological combinatorics [10], in particular to deep results involved in proof of the Lovász hypothesis [1].

A multihomomorphism $G \rightarrow H$ is a mapping $f: V_{G} \rightarrow 2^{V_{H}} \backslash\{\emptyset\}$ (i.e., associating a nonempty subset of vertices of $H$ with every vertex of $G$ ) such that whenever $\left\{u_{1}, u_{2}\right\}$ is an edge of $G$, we have $\left(v_{1}, v_{2}\right) \in E_{H}$ for every $v_{1} \in f\left(u_{1}\right)$ and every $v_{2} \in f\left(u_{2}\right)$.

The functions from vertices to sets can be seen as representation of relations. A relation with full domain thus can be regarded as surjective multihomomorphism, a multihomomorphism such that pre-image of every vertex in $H$ is non-empty and for every edge $(u, v)$ in $H$ we can find an edge $(x, y)$ in $G$ satisfying $u \in f(x), v \in f(y)$.

\subsection{Examples}

Similarly to graph homomorphisms, the equation $G * R=H$ (or $G \star R=H$ respectively) may have multiple solutions for some pairs of graphs $(G, H)$, while there may be no solution at all for other pairs.

As an example, consider $K_{2}$ (two vertices $x, y$ connected by an edge) and $C_{3}$ (a cycle of three vertices $u, v, w)$. Denote $R_{1}=\{(u, x),(v, y)\}, R_{2}=\{(v, x),(w, y)\}, R_{3}=$ $\{(w, x),(u, y)\}$, then it is easily seen that $C_{3} * R_{i}=K_{2}$ for each $1 \leq i \leq 3$, i.e. the equation $C_{3} * R=K_{2}$ has more than one solution.

On the other hand, there is no relation $R$ such that $K_{2} * R=C_{3}$. Otherwise, each vertex of $C_{3}$ is related to at most one vertex of $K_{2}$, since $C_{3}$ is loop free; hence there exists a vertex in $K_{2}$ which has no relation to at least two vertices in $C_{3}$, w.l.o.g., one can assume $(x, u),(x, v) \notin R$; then the definition of $*$ implies that there is no edge between $u$ and $v$, which causes a contradiction.

Because relations do not need to have full domain (unlike graph homomorphisms), there is always an relation from a graph $G$ to its induced subgraph $G[W]$.

Relations with full domain are not restricted to surjective homomorphisms. As a simple example, consider paths $P_{1}$ with vertex set $\{x, y\}$ and $P_{2}$ with vertex set $\{u, v, w\}$, respectively, and set $R=\{(x, u),(x, w),(y, v)\}$. One can easily verify $P_{1} * R=P_{2}$ by direct computation. Here, $R$ is not functional since $x$ has two images.

\subsection{Outline and main results}

This paper is organized as follows.

In section 2 the basic properties of the strong relations between graphs are compiled. It is shown that relations compose and every relation can be decomposed in a standard way into a surjective and an injective relation (Corollary 2.3). We discuss some structural properties of graph preserved by the relations.

Equivalence on the class of graphs induced by the existence of relations between graphs is the topic of section 3. We consider two forms: the strong relational equivalence, where relations are required to be reversible, and weak relational equivalence. Equivalence classes of strong relational equivalence are characterized in Theorem 3.8. To describe equivalence classes of the weak relational equivalence we introduce the notion of an R-core of a graph 
and show that it is in many ways similar to the more familiar construction of the graph core (Theorem 3.17). We explore in particular the differences between core and R-core and an effective algorithm to compute the R-core of given graph is provided.

Section 4 is concerned with the partial order induced on relations between two fixed graphs $G$ and $H$. Focusing on the special case $G=H$ the minimal elements of this partial order are described. In Theorem 4.7 we give a, perhaps surprisingly simple, characterization of those graphs $G$ for which all relations of $G$ to itself are automorphisms.

$\mathrm{R}$-retraction is defined in section 5 in analogy to retractions. It naturally gives rise to a notion of R-reduced graphs that we show to coincide with the concept of graph cores. By reversing the direction of relations, however, we obtain the concept of a cocore of a graph, which does not have a non-trivial counterpart in the world of ordinary graph homomorphisms, and explore its properties.

The computational complexity of testing for the existence of a relation between two graphs is briefly discussed in section 6. In Theorem 6.1 we describe the reduction of this problem to the surjective homomorphism problem.

Finally, in section 7 we briefly summarize the most important similarities and differences between weak and strong relational composition.

\section{Basic properties}

\subsection{Composition}

Recall that the composition of binary relations is associative, i.e., suppose $R \subseteq W \times X$, $S \subseteq X \times Y$, and $T \subseteq Y \times Z$. Then $R \circ(S \circ T)=(R \circ S) \circ T$. Furthermore, the transposition of relations satisfies $(R \circ S)^{+}=S^{+} \circ R^{+}$. Interpreting the graph $G$ as a relation on its vertex set, we easily derive the following identities:

Lemma 2.1 (Composition law). $(G * R) * S=G *(R \circ S)$.

Proof. $(G * R) * S=S^{+} \circ\left(R^{+} \circ G \circ R\right) \circ S=\left(S^{+} \circ R^{+}\right) \circ G \circ(R \circ S)$ $=(R \circ S)^{+} \circ G \circ(R \circ S)=G *(R \circ S)$.

Now we show that every relation $R$ can be decomposed, in a standard way, to a relation $R_{D}$ duplicating vertices and a relation $R_{C}$ contracting vertices.

Lemma 2.2. Let $R \subseteq X \times Y$ be a relation. Then there exists a subset $A$ of $X$, a set $B$, an injective relation with full domain $R_{D} \subseteq A \times B$ and a functional relation $R_{C} \subseteq B \times Y$, such that $R=I_{A} \circ R_{D} \circ R_{C}$, where $I_{A}$ is the identity on $X$ restricted to $A$.

Proof. Put $A=\operatorname{dom} R$. Then the relation $I_{A}$ removes vertices in $X \backslash \operatorname{dom} R$. It remains to show, therefore, that any relation $R \subseteq X \times Y$ with full domain can be decomposed into an injective relation $R_{D} \subseteq X \times B$ and a functional relation $R_{C} \subseteq B \times Y$. To see this, set $B=R$ and declare $(x, \alpha) \in R_{D}$ if and only if $\alpha=(x, p) \in R$ for some $p \in Y$, and $(\beta, q) \in R_{C}$ if and only if $\beta=(y, q) \in R$ for some $y \in X$. By construction $R_{D}$ is injective and $R_{C}$ is functional. Furthermore, $\left(x_{0}, p_{0}\right) \in R_{D} \circ R_{C}$ if and only if there is $\alpha \in R$ that is simultaneously of the form $\left(x_{0}, p\right)$ and $\left(x, p_{0}\right)$, i.e., $x=x_{0}$ and $p=p_{0}$. Hence $\left(x_{0}, p_{0}\right) \in R$.

Note that this decomposition is not unique. For instance, we could construct $B$ from multiple copies of $R$. More precisely, let $B=R \times\{1,2, \cdots, k\}$, then we would set $(x,(\alpha, i)) \in R_{D}(1 \leq i \leq k)$ if and only if $\alpha=(x, p) \in R$ for some $p \in Y$, etc. 
The set $B$ as constructed in the proof of Lemma 2.2 has minimal size. To see this, it suffices to show that, given $B$ there is a mapping from $B$ onto $R$. Since $R_{D}$ is injective and $R_{C}$ is functional we may set

$$
\alpha \in B \mapsto\left(R_{D}^{-1}(\alpha), R_{C}(\alpha)\right)
$$

Since $R=I_{A} \circ R_{D} \circ R_{C}$ we conclude that the mapping is surjective, and hence $|B| \geq|R|$.

According to Lemma 2.1, the decomposition of $R$ in Lemma 2.2 can be restated as follows:

Corollary 2.3. Suppose $G * R=H$. Then there is a set $B$, an injective relation $R_{D} \subseteq$ $\operatorname{dom} R \times B$ with full domain, and a surjective relation $R_{C} \subseteq B \times \operatorname{img} R$ such that $G[\operatorname{dom} R] * R_{D} * R_{C}=H$.

In diagram form, this is expressed as

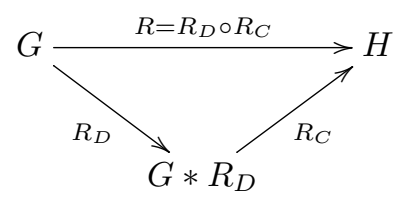

We shall remark that from the fact the relations compose it follows that the existence of a relation implies a quasi-order on graphs that is related to the homomorphism order. This order is studied more deeply in [8].

\subsection{Structural properties preserved by relations}

In this subsection we investigate structural properties of $H$ that can be derived from knowledge about certain properties of $G$ and the fact that there is some relation $R$ such that $G * R=H$.

\subsubsection{Connected components}

Proposition 2.4. Let $G * R=H$ and denote by $H_{1}, \cdots, H_{k}$ the connected components of $H$. Then there are relations $R_{i} \subseteq V_{G} \times V_{H_{i}}$ for each $1 \leq i \leq k$ such that $G * R_{i}=H_{i}$ and $R=\bigcup_{i=1}^{k} R_{i}$. Furthermore, set $G_{i}=G\left[R^{-1}\left(V_{H_{i}}\right)\right]$. Then there are no edges between $G_{i}$ and $G_{j}$ for arbitrary $i \neq j$.

Proof. Define the restriction of $R$ to the connected components of $H$ as $R_{i}=\{(x, y) \in$ $R \mid y \in V_{H_{i}}$. Clearly, $R$ is the disjoint union of the $R_{i}$ and $G * R_{i} \subseteq H_{i}$. The definition of * implies $H=G * R=\left(\bigcup_{i} R_{i}\right)^{+} \circ G \circ\left(\bigcup_{j} R_{j}\right)=\bigcup_{i} \bigcup_{j} R_{i}^{+} \circ G \circ R_{j}$. Since $R_{i}$ and $R_{j}$ relate vertices of $G$ to different connected components of $H$, we have $R_{i}^{+} \circ G \circ R_{j}=\emptyset$. It follows that $H=\bigcup_{i} \bigcup_{j} R_{i}^{+} \circ G \circ R_{j}=\bigcup_{i} R_{i}^{+} \circ G \circ R_{i}=\bigcup_{i} G * R_{i}$. Hence $G * R_{i}=H_{i}$.

Any edge between $G_{i}$ and $G_{j}$ would generate edges between $H_{i}$ and $H_{j}$, thus causing a contradiction to our assumptions.

Denote by $b_{0}(G)$ the number of connected components of $G$, then from Proposition 2.4 we arrive at: 
Corollary 2.5. Suppose both $G$ and $H$ do not have isolated vertices. If $G * R=H$ and $R$ has full domain, then $b_{0}(G) \geq b_{0}(H)$.

Proof. Our notations is the same as in Proposition 2.4. We claim for arbitrary connected component $C$ of graph $G$, there exists a unique $i$, such that $C$ is a connected component of $G_{i}$. Otherwise one can find two vertices $x, y \in C, x$ and $y$ adjacent, such that $x \in V_{G_{i}}$ and $y \in V_{G_{j}}$, since $G$ has no isolated vertices, which contradicts $E\left(G_{i}, G_{j}\right)=\emptyset$. Thus $b_{0}(G) \geq b_{0}(H)$ is easily followed.

From corollary 2.5, we know that $H$ is connected whenever $G$ is connected. The connectedness of $G$, however, cannot be deduced from the connectedness of $H$. For example, consider $G=P_{1} \cup P_{1}$ with vertex set $\left\{x_{1}, x_{2}, x_{3}, x_{4}\right\}$ and edges $\left\{x_{1}, x_{2}\right\}$ and $\left\{x_{3}, x_{4}\right\}$, and $H=P_{2}$ with vertex set $\left\{v_{1}, v_{2}, v_{3}\right\}$. Set $R=\left\{\left(x_{1}, v_{1}\right),\left(x_{2}, v_{2}\right),\left(x_{3}, v_{2}\right),\left(x_{4}, v_{3}\right)\right\}$. One can easily verify that $G * R=H$. On the other hand, $H$ is connected but $G$ has 2 connected components. The point here is, of course, that $R$ is not injective.

\subsubsection{Colorings}

Graph homomorphisms of simple graphs can be seen as generalizations of colorings: A (vertex) $k$-coloring of $G$ is a mapping $c: V_{G} \rightarrow\{1,2, \ldots, k\}$ such that adjacent vertices have distinct colors, i.e., $c(u) \neq c(v)$ whenever $(u, v) \in E_{G}$. Every $k$-coloring $c$ can be also seen as a homomorphism $c: G \rightarrow K_{k}$.

The chromatic number $\chi$ is defined as the minimal of colors needed for a coloring, see e.g. [7]. Thus, if $R$ is a functional relation describing a vertex coloring, then $G * R \subseteq K_{k}$. Conversely, $G * R \subseteq K_{k}$, where $R$ has full domain, then from Lemma 1.4, there exists a homomorphism from $G$ to $K_{k}$, which is a coloring of $G$.

Lemma 2.6. If $G$ is a simple graph and $R$ has full domain, then $\chi(G) \leq \chi(G * R)$.

Proof. Suppose $G * R=H$ and the domain of $R$ is full, from Lemma 1.4 we know $G \rightarrow H$, so $\chi(G) \leq \chi(G * R)$.

\subsubsection{Distances}

Observation 2.7. If $P_{k} * R=G, G$ is a simple graph and the domain of $R$ is full, $P_{k}$ with the vertex set $0,1, \cdots, k$, then there is a walk $\left[v_{0}, v_{1}, \ldots, v_{k}\right]$ in $G$, where $\left(i, v_{i}\right) \in R$ for $0 \leq i \leq k$.

Observation 2.8. If $C_{k} * R=G, G$ is a simple graph and the domain of $R$ is full, then there is a closed walk $\left[v_{0}, v_{1}, \ldots, v_{k-1}\right]$ in $G$, where $\left(i, v_{i}\right) \in R$ for $0 \leq i \leq k-1$.

Let $d_{G}(x, y)$ denote the canonical distance on graph $G$, i.e., $d_{G}(x, y)$ is the minimal length of a path in graph $G$ that connects vertices $x$ and $y$; if there is no path connects vertices $x$ and $y$, then the distance is infinite.

Lemma 2.9. Suppose there exists a relation $R$ with full domain s.t. $G * R=H, x, y \in V_{G}$, $u, v \in V_{H}$ and $(x, u) \in R,(y, v) \in R$. If $x \neq y$, then $d_{H}(u, v) \leq d_{G}(x, y)$; If $x=y$ and $x$ is not an isolated vertex, then $d_{H}(u, v) \leq 2$. 
Proof. If $x=y$ and $x$ is not isolated, pick a vertex $z$ of graph $G$ which is adjacent to vertex $x$, and find a vertex $w \in H$ satisfying $(z, w) \in R$. Then $(w, u) \in E_{H}$ and similarly $(w, v) \in E_{H}$. So $d_{H}(u, v) \leq 2$.

If $x \neq y$, choose the shortest path $P=x, x_{1}, x_{2}, \cdots, x_{k}, y$ between $x$ and $y$, and find corresponding vertices $u_{1}, u_{2}, \cdots, u_{k} \in H$ such that $\left(x_{i}, u_{i}\right) \in R$ for any $1 \leq i \leq k-1$ it is easily seen that $\left(u, u_{1}\right) \in E_{H},\left(u_{i}, u_{i+1}\right) \in E_{H}$ and $\left(u_{k}, v\right) \in E_{H}$, then $d(u, v) \leq$ $d(x, y)$.

The eccentricity $\epsilon$ of a vertex $v$ is the greatest distance between $v$ and any other vertex. The radius of a graph $G$, denoted by $\operatorname{rad}(G)$, is the minimum eccentricity of any vertex. The diameter of a graph $G$, denoted by $\operatorname{diam}(G)$, is the maximum eccentricity of any vertex in the graph, i.e., the largest distance between any pair of vertices.

Corollary 2.10. Suppose $G * R=H, G$ and $H$ are connected graphs, and $R$ has full domain, then $\operatorname{rad}(H) \leq \max \{\operatorname{rad}(G), 2\}$.

An analogous result holds for the diameters. In particular, if $G$ is not a complete graph, then $\operatorname{diam}(G) \geq \operatorname{diam}(G * R)$.

Corollary 2.11. There is a relation from the path of length $k, P_{k}$, to the path of length $l$, $P_{l}$, if and only if either $k \geq l$ or $k=1, l=2$.

Proof. For $k \geq l$ there is a surjective homomorphism $f$ from $P_{k}$ to $P_{l}$ and hence by Lemma 1.4 there is also a relation from $P_{k}$ to $P_{l}$. In Section 1.5 we already showed a relation from $P_{1}$ to $P_{2}$.

To show that $P_{1} * R=P_{2}$ is the only case with $k<l$ we first observe that Lemma 2.9 excludes the existence of relation from $P_{k}$ to $P_{l}$ for $1<k<l$. Now suppose $R$ satisfies $P_{1} * R=P_{k}$ for $k>2$. Since $P_{k}$ has at least 4 vertices, either one of the vertices of $P_{1}$ has at least 3 images so that $P_{1} * R$ has a vertex with degree at least 3 , or both of the vertices in $P_{1}$ have at least 2 images, in which case all vertices of $P_{1} * R$ have degree at least 2 . In both cases $P_{1} * R$ cannot be a path.

In particular, $\left\{P_{1}, P_{2}\right\}$ is the only pair of paths such that there is a relation between them in both directions.

\subsubsection{Complete graphs}

The complement graph $\bar{H}$ of a simple graph $H$ has the same vertex set as $H$, and two vertices are connected in $\bar{H}$ if and only if they are not connected in $H$.

Note that in this subsection we do not require that the domain of $R$ is full.

Proposition 2.12. Let $H$ be a simple graph. Then there exists a relation $R$ such that $K_{k} * R=H$ if and only if $\bar{H}$ is the disjoint union of at most $k$ complete graphs.

Proof. Denote the connected components of $\bar{H}$ by $H_{1}, \ldots, H_{m}$. If $m \leq k$ and every connected component of $\bar{H}$ is a complete graph, let $R=\left\{(i, u) \mid i=1, \cdots, m, u \in V_{H_{i}}\right\}$ and by the definition of complement graph, for any $i=1, \cdots, m$, all the vertices in $H_{i}$ are independent in $H$, and $u$ is adjacent to $v$ whenever $u \in V_{H_{i}}$ and $v \in V_{H_{j}}$ for distinct $i, j$. Hence it is easily seen that $K_{k} * R=H$.

Conversely, if $K_{k} * R=H$, denote the vertices in $K_{k}$ by $1, \cdots, k$, s.t. $\operatorname{dom} R=$ $\{1, \cdots, m\}$. We claim that $R$ is injective, otherwise $H$ would have loops. Thus $V_{H}$ is 
the disjoint union of $R(1), \cdots, R(m)$. For any two distinct vertices $u, v$ in $R(i), u$ and $v$ are independent in $H$ and for distinct $i$ and $j$ every vertex in $R(i)$ are adjacent with every vertex in $R(j)$ whenever $R(i) \neq \emptyset$. Therefore for any $i, R(i)$ is the vertex set of a connect component of $\bar{H}$, which is a complete graph.

\subsubsection{Subgraphs}

Relations between graphs intuitively imply relations between local subgraphs. In this section we make this concept more precise. Denote by

$$
N_{G}[x]:=\left\{z \in V_{G} \mid z=x \vee(x, z) \in E_{G}\right\}
$$

the closed neighborhood of $x$ in $G$. Furthermore, we let $\overline{N_{G}[x]}:=V_{G} \backslash N_{G}[x]$ be the set of vertices that are not adjacent (or identical) to $x$ in $G$ and denote by $\overline{G_{x}}:=G\left[\overline{N_{G}[x]}\right]$ the induced subgraph of $G$ that is obtained by removing the closed neighborhood of a vertex $x$.

Analogously, for a subset $S \subseteq V_{G}$ we define

$$
\bar{S}=G\left[V_{G} \backslash \bigcup_{x \in S} N_{G}[x]\right]
$$

as the induced subgraph obtained by removing all vertices in $S$ and their neighbors.

Then we have the following result about relations between local subgraphs.

Proposition 2.13. Suppose $G * R=H$ and $S$ and $D$ are subsets of $V_{G}$ and $V_{H}$, respectively, such that $\left.G[S] * R\right|_{(S \times D)}=H[D],\left.R\right|_{(S \times D)}$ has full domain on $S$, and there is no isolated vertex in $\bar{D}$. Then $\bar{S} * \tilde{R}=\bar{D}$, where $\tilde{R}=\left.R\right|_{(\bar{S} \times \bar{D})}$ is the corresponding restriction of $R$.

Proof. Obviously, $\bar{S} * \tilde{R}$ is an induced subgraph of $\bar{D}$. We have to show the reverse inclusion. Given $u \in V_{\bar{D}}$ and $x \in R^{-1}(u)$, we first show that there are two possibilities:

1. $x$ is a vertex of $\bar{S}$.

2. $x$ is an isolated vertex of $S$.

Assume that is not the case, i.e., that $x \notin V_{\bar{S}}$ and that $x$ is either a non-isolated vertex of $S$ or $x$ is in the neighborhood of some vertex of $S$. In either case there is $y \in S$ connected by an edge to $x$. Consequently there is also $v \in D$, such that $v \in R(y)$, connected by an edge to $u$. It follows $u \notin V_{\bar{D}}$, a contradiction.

Now consider an arbitrary edge $(u, v) \in E_{\bar{D}}$. We have $(x, y) \in E_{G}$ such that $u \in R(x)$ and $v \in R(y)$. It follows that $x$ and $y$ are not isolated and thus $x, y$ are vertices of $\bar{S}$. Consequently $\bar{S} * \tilde{R}$ has precisely the same edges as $\bar{D}$. Because $\bar{D}$ has no isolated vertices and thus every vertex is an endpoint of some edge, we know that the vertex set of $\bar{S} * \tilde{R}$ is same as the vertex set of $\bar{D}$.

This result is of particular practical use in the special case where $S$ and $D$ consist of a single vertex. When looking for a relation $R$ such that $G * R=H$ one can remove a vertex including its neighborhood from $G$ as well as the prospective image including the neighborhood from $H$ and solve the problem on the subgraphs. 


\section{Relational equivalence}

Graphs $G$ and $H$ are homomorphism equivalent (or hom-equivalent) if there exists homomorphisms $G \rightarrow H$ and $H \rightarrow G$. It is well known that every equivalence class of the homomorphism order contains a minimal representative that is unique up to isomorphism: the graph core [7].

We define similar equivalences implied by the existence of (special) relations between graphs. In this section, we require all relations to have full domain unless explicitly stated otherwise. With this condition we will show that these equivalences produce a rich structure closely related to but distinct from the structure of homomorphism equivalences.

This may come as a surprise: the equivalence implied by the existence of surjective homomorphisms is not interesting. Consider two graphs $G$ and $H$ and suppose there are surjective homomorphisms $f: G \rightarrow H$ and $g: H \rightarrow G$. Since every vertex in $V_{G}$ has at most one image under $f$, we have $\left|V_{G}\right| \geq\left|V_{H}\right|$. Analogously $\left|V_{H}\right| \geq\left|V_{G}\right|$, and hence $\left|V_{G}\right|=\left|V_{H}\right|$. Thus $f$ and $g$ are both bijective, and $G$ is isomorphic to $H$.

\subsection{Reversible relations}

Definition 3.1. A relation $R$ is reversible with respect to graph $G$ if $(G * R) * R^{+}=G$.

We write $N_{G}(x):=\left\{z \in V_{G} \mid(x, z) \in E_{G}\right\}$ for the open neighborhood of vertex $x$ in graph $G$.

Proposition 3.2. Suppose $R=R_{D} \circ R_{C}$, where $R_{D}$ and $R_{C}$ are constructed as in the proof of Proposition 2.2. Then $R$ is reversible with respect $G$ if and only if for every $\alpha$ and $\beta$ satisfying $R_{C}(\alpha)=R_{C}(\beta)$ we have $N_{G * R_{D}}(\alpha)=N_{G * R_{D}}(\beta)$.

Proof. We set $G_{1}=G * R_{D}$, then from Lemma 2.1 we have $G_{1} * R_{C}=H$. If $R_{C}(\alpha)=$ $R_{C}(\beta)$ implies $N_{G_{1}}(\alpha)=N_{G_{1}}(\beta)$, then $H * R_{C}^{+}=G_{1}$. Since $G_{1} * R_{D}^{+}=H$, we have $H * R_{C}^{+} * R_{D}^{+}=H * R^{+}=G$, i.e., $R$ is reversible.

Conversely, since $R$ is reversible, i.e., $H * R^{+}=G$, setting $G_{2}=H * R_{C}^{+}$gives $G_{2} * R_{D}^{+}=G$. Hence $G_{1} * R_{C} * R_{C}^{+}=G_{2}$ and $G_{2} * R_{D}^{+} * R_{D}=G_{1}$. From $I_{G_{1}} \subseteq R_{C} * R_{C}^{+}$ we conclude $G_{1} \subseteq G_{2}$, and similarly $I_{G_{2}} \subseteq R_{D}^{+} * R_{D}$ yields $G_{1} \supseteq G_{2}$. Hence $G_{1}=G_{2}$. $R_{C}^{+}$is injective, hence $\alpha, \beta \in V_{G_{2}}=V_{G_{1}}$ has the same open neighborhood whenever the pre-image of $\alpha$ and $\beta$ under $R_{C}^{+}$coincide, i.e. $R_{C}(\alpha)=R_{C}(\beta)$.

$R_{D}$ is an injective relation, hence one can easily get $N_{G * R_{D}}(\alpha)=R_{D}\left(N_{G}(x)\right)$ provided that $(x, \alpha) \in R_{D}$. On the other hand, if we define $R$ to be the image of $R_{D}$ as in the proof of Proposition 2.2, then $R_{C}(\alpha)=R_{C}(\beta)$ implies there are two distinct vertices $x, y \in V_{G}$, s.t. $(x, u),(y, u) \in R$, where $u=R_{C}(\alpha)=R_{C}(\beta)$, and verse visa. Using Proposition 3.2 we thus obtain

Proposition 3.3. A relation $R$ is reversible with respect to $G$ if and only if for every two vertices $x$ and $y$ such that $R(x) \cap R(y) \neq \emptyset$ we have $N_{G}(x)=N_{G}(y)$.

\subsection{Strong relational equivalence}

Definition 3.4. Two graphs $G$ and $H$ are (strongly) relationally equivalent, $G \backsim H$, if there is a relation $R$ such that $G * R=H$ and $H * R^{+}=G$.

Lemma 3.5. Relational equivalence is an equivalence relation on graphs. 


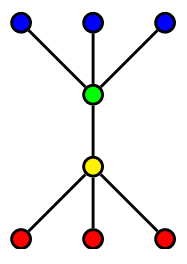

G

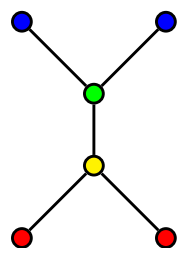

$H$

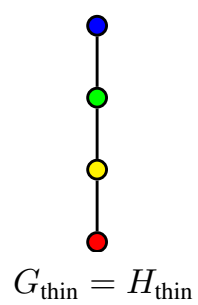

Figure 2: Non-isomorphic graphs $G$ and $H$ with isomorphic thin graphs.

Proof. The relation $\backsim$ is reflexive since $G * I_{G}=G$. Symmetry also follows directly from the definition. Suppose $G * R=H$ and $H * R^{+}=G$ and $H * Q=K$ and $K * Q^{+}=H$, i.e., $(G * R) * Q=K$ and $\left(K * Q^{+}\right) * R^{+}=G$, i.e., $G *(R \circ Q)=K$ and $K *\left(Q^{+} \circ R^{+}\right)=K *(R \circ Q)^{+}=G$, i.e., $\sim$ is also transitive.

Definition 3.6. The thinness relation $S$ of $G$ is the equivalence relation on $V_{G}$ defined by $(x, y) \in S$ if and only if $N_{G}(x)=N_{G}(y)$. A graph $G$ is called thin if every vertex forms its own class in $S$.

Thin graphs are also known as "point determining graphs" [13].

We denote by $\mathcal{S}$ the corresponding partition of $V_{G}$, and write $R_{S} \subseteq V_{G} \times \mathcal{S}$ for the relation that associates each vertex with its $S$-equivalence class, i.e., $(x, \beta) \in R_{S}$ if and only if $x \in \beta$.

Definition 3.7. The thin graph of $G$, denoted by $G_{\text {thin }}$, is the quotient graph $G / S$, i.e., $G_{\text {thin }}$ has vertex set $\mathcal{S}$ and two equivalence classes $\sigma$ and $\tau$ of $S$ are adjacent in $G_{\text {thin }}$ if and only if $(x, y)$ is an edge of $G$ with $x \in \sigma$ and $y \in \tau$.

As noted e.g. in [6, p.81], $G_{\text {thin }}$ is itself a thin graph. Furthermore, $R_{S}$ is a full homomorphism of $G$ to $G_{\text {thin }}$, see [4].

Thinness and the quotients w.r.t. the thinness relation play an important role in particular in the context of product graphs, see [9]. In this context it is well known that $G$ can be reconstructed from $G_{\text {thin }}$ and the knowledge of the $S$-equivalence classes. In fact, we have

$$
G_{\text {thin }} * R_{S}^{+}=G \text {. }
$$

Theorem 3.8. $G$ and $H$ are in the same equivalence class w.r.t. $\backsim$ if and only if their thin graphs are isomorphic.

Proof. Assume $G \backsim H$. From Equation(3.1) we know that $G \backsim G_{\text {thin }}, H \backsim H_{\text {thin }}$, so $G_{\text {thin }} \backsim H_{\text {thin }}$. Now we claim that $G_{\text {thin }}$ and $H_{\text {thin }}$ are isomorphic. Suppose $G_{\text {thin }} * R=H_{\text {thin }}$, then the pre-image of $R$ is unique. Otherwise, there exist distinct vertices $x, y \in V_{G_{\text {thin }}}$ such that $R(x)=R(y)$, then $N_{G_{\text {thin }}}(x)=N_{G_{\text {thin }}}(y)$, contradicting thinness. Likewise, the preimage of $R^{-1}$ is unique, i.e., the image of $R$ is unique. Hence $R$ is one-to-one.

The example in Fig. 2 shows that thin graphs can be isomorphic while $G$ and $H$ themselves are not isomorphic. Relational equivalence thus is coarser than graph isomorphism (surjective homomorphic equivalence) but stronger than homomorphic equivalence. 

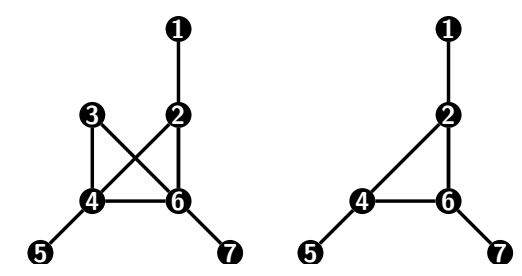

Figure 3: $G$ and $H$ are weakly relationally equivalent but have non-isomorphic thin graphs.

\subsection{Weak relational equivalence}

Definition 3.9. Two graphs $G$ and $H$ are weak relationally equivalent, $G \sim_{w} H$, if there are relations $R$ and $S$ such that $G * R=H$ and $H * S=G$.

Lemma 3.10. Weak relational equivalence is an equivalence relation on graphs.

Proof. By definition $\sim_{w}$ is symmetric. Because $G * I_{G}=G$, relation $\sim_{w}$ is reflexive. Suppose $G \sim_{w} G^{\prime}$ and $G^{\prime} \sim_{w} G^{\prime \prime}$. Thus there are relations $R, S, R^{\prime}$, and $S^{\prime}$, such that $G^{\prime}=G * R, G^{\prime \prime}=G^{\prime} * R^{\prime}, G=G^{\prime} * S$, and $G^{\prime}=G^{\prime \prime} * S^{\prime}$. By the composition law (Lemma 2.1) it follows that $G^{\prime \prime}=G *\left(R \circ R^{\prime}\right)$ and $G=G^{\prime \prime} *\left(S^{\prime} \circ S\right)$, i.e, $G \sim_{w} G^{\prime \prime}$. Hence $\sim_{w}$ is transitive.

Strong relational equivalence implies weak relational equivalence. To see this, simply observe that the definition of the weak form is obtained from the strong one by setting $S=R^{+}$.

The converse is not true, as shown by the graphs $G$ and $H$ in Fig. 3: It is easy to see that their thin graphs are different and thus $G$ and $H$ are not strongly relationally equivalent. However, are relationally equivalent. To get relation from $G$ to $H$ contract vertices 2 and 3 and keep other vertices on place, i.e.,

$$
R=\{(1,1),(2,2),(3,2),(4,4),(5,5),(6,6),(7,7)\}
$$

To get relation from $H$ to $G$, duplicate 5 and 7 and contract them together to 3 ,

$$
S=\{(1,1),(2,2),(4,4),(5,5),(6,6),(7,7),(5,3),(7,3)\}
$$

Consequently, weak relational equivalence is coarser than strong relational equivalence.

\subsection{R-cores}

A graph is an $R$-core, if it is the smallest graph (in the number of vertices) in its equivalence of $\sim w$.

This notion is analogous to the definition of graph cores. In this section we show properties of R-cores that are similar to the properties of graph cores. To this end we first need to develop a simple characterization of R-cores.

Again we start from a decomposition of relations. Consider a relation $R$ such that $G * R=H$. We seek for pair of relations $R_{1}$ and $R_{2}$ such that $R=R_{1} \circ R_{2}$. In contrast 
to Lemma 2.2, however, we now look for a decomposition so that the graph $G^{\prime}=G * R_{1}$ is smaller (in the number of vertices) than $G$.

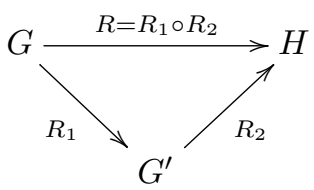

The existence of such a decomposition follows from a translation of the well-known Hall Marriage Theorem [12] to the language of relations. We say that the relation $R \subseteq A \times B$ satisfies the Hall condition, if for every $S \subseteq A$ we have $|S| \leq|R(S)|$.

Theorem 3.11 (Hall's theorem). If $G * R=H$ and $R$ satisfies the Hall condition, then $R$ contains a monomorphism $f: G \rightarrow H$.

Proof. The Hall Marriage Theorem is usually described on set systems. For set systems satisfying the Hall condition, the theorem guarantees the existence of a system of distinct representatives, see i.e. [12]. Relations can be seen as set systems (defined by the images of individual vertices). Furthermore, in our setting the system of distinct representatives directly corresponds to a monomorphism contained in the relation $R$.

Lemma 3.12. If $G * R=H$ and relation $R$ does not satisfy the Hall condition, then there are relations $R_{1}$ and $R_{2}$ such that $R=R_{1} \circ R_{2}$, and the number of vertices of graph $G^{\prime}=G * R_{1}$ is strictly smaller than the number of vertices of $G$.

Proof. Without loss of generality assume that $V_{G} \cap V_{H}=\emptyset$. If $R$ does not satisfy the Hall condition, then there exists a vertex set $S \subset V_{G}$ such that $|S|>|R(S)|$. Now we define relations $R_{1}$ and $R_{2}$ as follows:

$$
R_{1}(x)=\left\{\begin{array}{l}
R(x) \text { for } x \in S, \\
x \text { otherwise, }
\end{array} \quad R_{2}(x)=\left\{\begin{array}{l}
x \text { for } x \in R(S), \\
R(x) \text { otherwise }
\end{array}\right.\right.
$$

Obviously $R_{1} \circ R_{2}=R$ and $\left|V_{G^{\prime}}\right|=\left|V_{G}\right|-(|S|-|R(S)|)<\left|V_{G}\right|$.

This immediately gives a necessary, but in general not sufficient, condition for a graph to be an R-core.

Corollary 3.13. If $G$ is an $R$-core, then every relation $R$ such that $G * R=G$ satisfies the Hall condition and thus contains a monomorphism.

Proof. Assume that there is a relation $R$ that does not satisfy the Hall condition. Then there is a graph $G^{\prime},\left|V_{G^{\prime}}\right|<\left|V_{G}\right|$, and relations $R_{1}$ and $R_{2}$ such that $G * R_{1}=G^{\prime}$ and $G^{\prime} * R_{2}=G$. Consequently $G^{\prime}$ is a smaller representative of the equivalence class of $\sim_{w}$, a contradiction with $G$ being R-core.

To see that the condition of Corollary 3.13 is not sufficient consider a graph consisting of two independent vertices.

Next we show that R-cores are, up to isomorphism, unique representatives of the equivalence classes of $\sim$. 


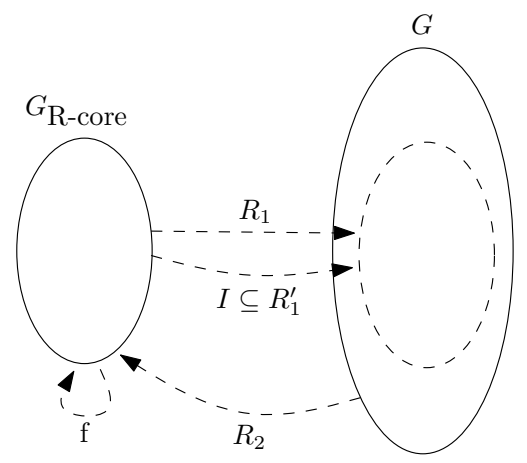

Figure 4: Construction of an embedding from $G_{\mathrm{R} \text {-core }}$ to $G$.

Proposition 3.14. If both $G$ and $H$ are R-cores in the same equivalence class of $\sim_{w}$, then $G$ and $H$ are isomorphic.

Proof. Because both $G$ and $H$ are R-cores, we know that $\left|V_{G}\right|=\left|V_{H}\right|$.

Consider relations $R_{1}$ and $R_{2}$ such that $G * R_{1}=H$ and $H * R_{2}=G$. Applying Lemma 3.12 we know that $R_{1}$ satisfies the Hall condition. Otherwise there would be a graph $G^{\prime}$ with $\left|V_{G^{\prime}}\right|<\left|V_{G}\right|$ so that $G^{\prime}$ is relationally equivalent to both $G$ and $H$ contradicting the fact that $G$ and $H$ are R-cores. Similarly, we can show that $R_{2}$ also satisfies the Hall condition.

From Theorem 3.11 we know that there is a monomorphism $f$ from $G$ to $H$, and monomorphism $g$ from $H$ to $G$. It follows that number of edges of $G$ is not larger than the number of edges of $H$ and vice versa. Because $G$ and $H$ have the same number of edges and same number of vertices, $G$ and $H$ must be isomorphisms.

It thus makes sense to define a construction analogous to the core of a graph.

Definition 3.15. $H$ is an $R$-core of graph $G$ if $H$ is an R-core and $H \sim_{w} G$.

All R-cores of graph $G$ are isomorphic as an immediate consequence of Prop. 3.14. We denote the (up to isomorphism) unique R-core of graph $G$ by $G_{\mathrm{R} \text {-core }}$.

Lemma 3.16. $G_{\mathrm{R} \text {-core }}$ is isomorphic to a (not necessarily induced) subgraph of $G$.

Proof. Take any relation $R$ such that $G_{\mathrm{R} \text {-core }} * R=G$. By the same argument as in Corollary 3.13 , there is a monomorphism $f: G_{\mathrm{R} \text {-core }} \rightarrow G$ contained in $R$. Consider the image of $f$ on $G$.

Theorem 3.17. $G_{R \text {-core }}$ is isomorphic to an induced subgraph of $G$.

Proof. Fix $R_{1}$ and $R_{2}$ such that $G_{\mathrm{R} \text {-core }} * R_{1}=G$ and $G * R_{2}=G_{\mathrm{R} \text {-core }}$.

$R=R_{1} \circ R_{2}$ is a relation such that $G_{\mathrm{R} \text {-core }} * R=G_{\mathrm{R} \text {-core }}$. By Corollary $3.13, R$ contains a monomorphism $f: G_{\mathrm{R} \text {-core }} \rightarrow G_{\mathrm{R} \text {-core }}$. Because such a monomorphism is a permutation, there exists $n$ such that $f^{n}$, the $n$-fold composition of $f$ with itself, is the identity. 
Put $R_{1}^{\prime}=R^{n-1} \circ R_{1}$. Because $R^{n}$ contains the identity and $R^{n}=R_{1}^{\prime} \circ R_{2}$, it follows that for every $x \in V_{G_{\mathrm{R}-\text { core }}}$, there is a vertex $I(x) \in V_{G}$ such that $I(x) \in R_{1}^{\prime}(x)$ and $x \in R_{2}(I(x))$.

We show that for two vertices $x \neq y$, we have $I(x) \neq I(y)$ and thus both $I$ and $I^{-1}$ are monomorphisms. Assume, that is not the case, i.e., that there are two vertices $x \neq y$ such that $I(x)=I(y)$. Consider an arbitrary vertex $z$ in the neighborhood of $x$. It follows that $I(z)$ must be in the neighborhood of $I(x)$ and consequently $z$ is in the neighborhood of $y$. Thus the neighborhoods of $x$ and $y$ are the same. By Theorem 3.8, however, we know that the R-core is a thin graph (because weak relational equivalence is coarser than strong relational equivalence), a contradiction.

Finally observe that $I$ is an embedding from $G_{\mathrm{R} \text {-core }}$ to $G$. For every edge $(x, y) \in$ $E_{G_{\mathrm{R}-\text { core }}}$ we also have edge $(I(x), I(y)) \in E_{G}$ because $I$ is contained in relation $R_{1}^{\prime}$. Similarly because $I^{-1}$ is contained in relation $R_{2}$, every edge $(I(x), I(y)) \in E_{G}$ corresponds to an edge $(x, y) \in E_{G_{\mathrm{R} \text {-oore }}}$.

We close the section with an algorithm computing the R-core of a graph. In contrast to graph cores, where the computation is known to be NP-complete, there is a simple polynomial algorithm for R-cores.

Observe that the R-core of a graph containing isolated vertices is isomorphic to the disjoint union of the R-core of the same graph with the isolated vertices removed and a single isolated vertex. The R-core of a graph without isolated vertices can be computed by Algorithm 1.

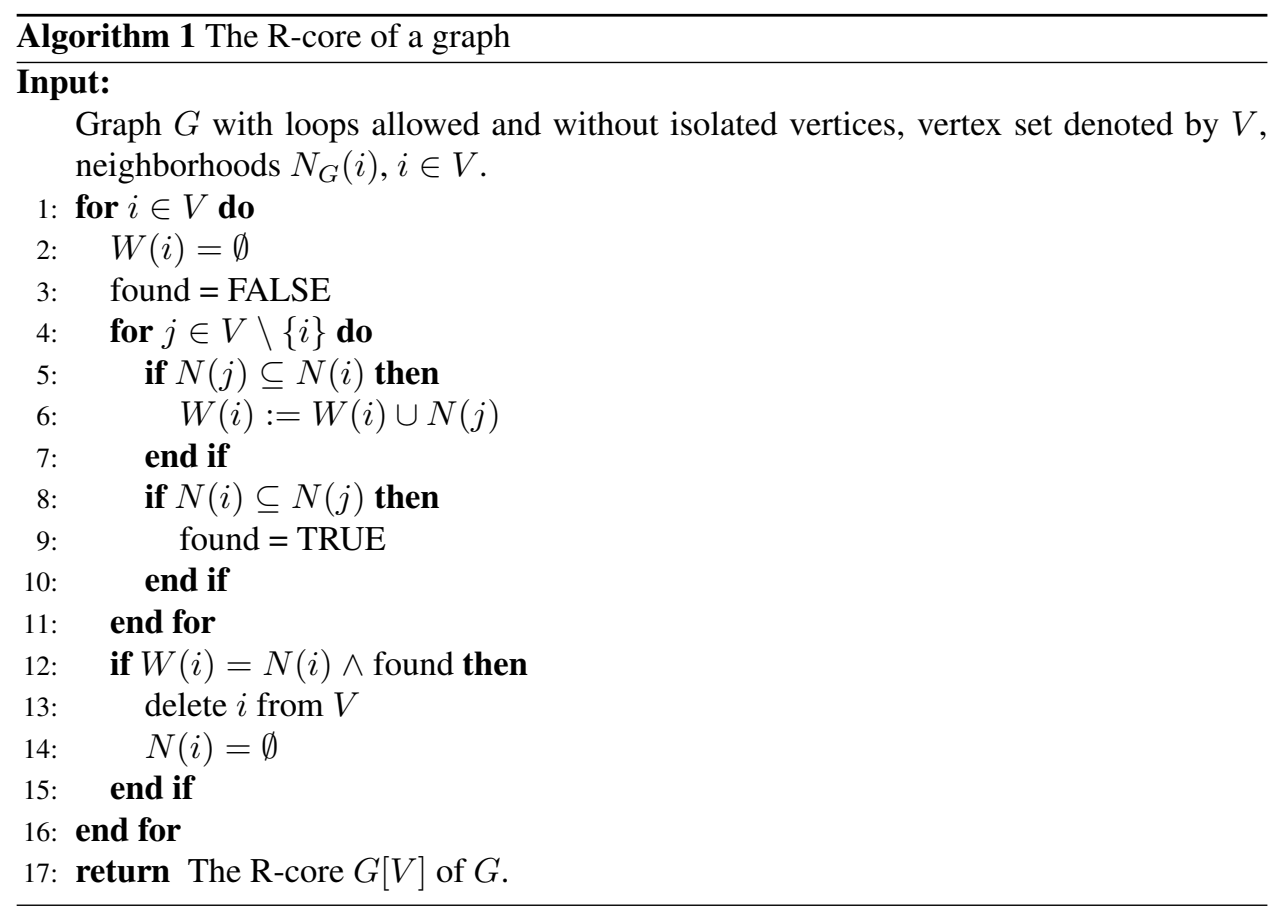

The algorithm removes all vertices $v \in G$ such that (1) the neighborhood of $v$ is union of neighborhood of some other vertices $v_{1}, v_{2}, \ldots, v_{n}$ and (2) there is vertex $u$ such that 
$N_{G}(v) \subseteq N_{G}(u)$

It is easy to see that the resulting graph $H$ is relationally equivalent to $G$. Condition (1) ensures the existence of a relation $R_{1}$ such that $H * R_{1}=G$, while the condition (2) ensures the existence of a relation $R_{2}$ such that $G * R_{2}=H$.

We need to show that $H$ is isomorphic to $G_{\mathrm{R} \text {-core }}$. By Theorem 3.17 we can assume that $G_{\mathrm{R}-c o r e}$ is an induced subgraph of $H$ that is constructed as an induced subgraph of $G$.

We also know that there are relations $R_{1}$ and $R_{2}$ such that $G_{\mathrm{R} \text {-core }} * R_{1}=H$ and $G * R_{2}=G_{\mathrm{R} \text {-core }}$. By the same argument as in the proof of Theorem 3.17 we can assume both $R_{1}$ and $R_{2}$ to contain an (restriction of) identity.

Now assume that there is a vertex $v \in V_{H} \backslash V_{G_{\mathrm{R}-\text { core }}}$. We can put $u=R_{2}(v)$ and because $R_{2}$ contains an identity we have $N_{G}(v) \subseteq N_{G}(u)$. We can also put $\left\{v_{1}, v_{2} \ldots v_{n}\right\}$ to be set of all vertices such that $v \in R_{1}\left(v_{i}\right)$. It follows that the neighborhood of $v$ is the union of neighborhoods of $v_{1}, v_{2}, \ldots, v_{n}$ and consequently we have $v \notin V_{H}$, a contradiction.

\section{The partial order $\operatorname{Rel}(G, H)$}

\subsection{Basic properties}

For fixed graphs $G$ and $H$ we consider partial order $\operatorname{Rel}(G, H)$. The vertices of this partial order are all relations $R$ such that $G * R=H$. We put $R_{1} \leq R_{2}$ if and only if $R_{1} \subseteq R_{2}$.

This definition is motivated by Hom-complexes, see [10]. In this section we show the basic properties of this partial order and concentrate on minimal elements in the special case of $\operatorname{Rel}(G, G)$.

Proposition 4.1. Suppose $G * R^{\prime}=H, G * R^{\prime \prime}=H$ and $R^{\prime} \subseteq R^{\prime \prime}$, then any relation $R$ with $R^{\prime} \subseteq R \subseteq R^{\prime \prime}$ also satisfies $G * R=H$.

Proof. From $R^{\prime} \subseteq R \subseteq R^{\prime \prime}$ we conclude $G * R^{\prime} \subseteq G * R \subseteq G * R^{\prime \prime}$. Hence $G * R^{\prime}=G * R^{\prime \prime}$ implies $G * R=H$.

Hence it is possible to describe the partial order $\operatorname{Rel}(G, H)$ by listing minimal and maximal solutions $R$ of $G * R=H$ w.r.t. set inclusion.

For example, if $G$ is $P_{3}$ with vertices $v_{0}, v_{1}, v_{2}, v_{3}$ and $H$ is $P_{1}$ with vertices $x_{0}, x_{1}$, it is easily seen that $R^{\prime \prime}=\left\{\left(v_{0}, x_{0}\right),\left(v_{2}, x_{0}\right),\left(v_{1}, x_{1}\right),\left(v_{3}, x_{1}\right)\right\}$ is a maximal solution of $G * R=H$ and $R^{\prime}=\left\{\left(v_{0}, x_{0}\right),\left(v_{1}, x_{1}\right)\right\}$ is a minimal solution, because $R^{\prime} \subset R^{\prime \prime}$, then all the relations $R$ with $R^{\prime} \subseteq R \subseteq R^{\prime \prime}$ satisfy $G * R=H$. We note that minimal and maximal solutions need not be unique.

\subsection{Solutions of $G * R=G$}

For simplicity, we say that a relation $R$ is an automorphism of $G$ if it is of the form $R=$ $\left\{(x, f(x)) \mid x \in V_{G}\right\}$ and $f: V_{G} \rightarrow V_{G}$ is an automorphism of $G$.

We shall see that conditions related to thinness again play a major role in this context. Recall that $G$ is thin if no two vertices have the same neighborhood, i.e., $N_{G}(x)=N_{G}(y)$ implies $x=y$. Here we need an even stronger condition:

Definition 4.2. A graph $G$ satisfies condition $N$ if $N_{G}(x) \subseteq N_{G}(y)$ implies $x=y$.

In particular, graph satisfying condition $\mathrm{N}$ is thin. 
Proposition 4.3. For a given graph $G$, the set $\operatorname{Rel}(G, G)$ of all relations satisfying $G * R=$ $G$ forms a monoid.

Proof. Firstly, because $G$ is a finite graph, the set $\operatorname{Rel}(G, G)$ is also finite. Furthermore, $R, S \in \operatorname{Rel}(G, G)$ implies $G * R=G$ and $G * S=G$ and thus $G *(R \circ S)=G$, so that $R \circ S \in \operatorname{Rel}(G, G)$. Finally, the identity relation $I_{G}$ is a left and right identity for relational composition: $I_{G} \circ R=R \circ I_{G}=R$.

A relation $R \subset V_{G} \times V_{G}$ can be interpreted as a directed graph $\vec{R}$ with vertex set $V_{G}$ and a directed edge $u \rightarrow v$ if and only if $(u, v) \in R$. Note that $\vec{R}$ may have loops. We say that $v \in V_{G}$ is recurrent if and only if there exists a walk (of length at least 1) from $v$ to itself. Let $S_{G}$ be the set of all the recurrent vertices. Furthermore, we define an equivalence relation $\xi$ on $S_{G}$ by setting $(u, v) \in \xi$ if there is a walk in $\vec{R}$ from $u$ to $v$ and vice versa. The equivalence classes w.r.t. $\xi$ are denoted by $\vec{R} / \xi=\left\{D_{1}, D_{2}, \cdots, D_{m}\right\}$. We furthermore define a binary relation $\geq$ over $\vec{R} / \xi$ as follows: if there is a walk from a vertex $u$ in $D_{i}$ to a vertex $v$ in $D_{j}$, then we say $u \geq v$. It is easily seen that $\geq$ is reflexive, antisymmetric, and transitive, hence $(\vec{R} / \xi, \geq)$ is a partially ordered set. W.l.o.g. we can assume $\left\{D_{1}, D_{2}, \ldots, D_{s}\right\}$ are the maximal elements w.r.t. $\geq$. Now let $G_{r}=G\left[D_{1} \cup \cdots \cup\right.$ $D_{s}$ ] be the subgraph of $G$ induced by these maximal elements.

In the following we write $R^{l}$ for the $l$-fold composition of $R$ with itself.

Lemma 4.4. For arbitrary $x \in V_{G}$, there exists $l \in \mathbb{N}$ and a recurrent vertex $v$ such that $(v, x) \in R^{l}$.

Proof. Set $x_{0}=x$ and choose $x_{i} \in R^{-1}\left(x_{i-1}\right)$ for all $i \geq 1$. Since $\left|V_{G}\right|<\infty$, there are indices $j, k \in \mathbb{N}, j<k, x_{j}=x_{k}$. Then $x_{j}$ is recurrent vertex. The lemma follows by setting $l=j$ and $v=x_{i}$.

Lemma 4.5. For every $v \in V_{G_{r}}, R^{-1}(v) \subseteq V_{G_{r}}$.

Proof. Suppose $x \in R^{-1}(v)$ is not recurrent. Lemma 4.4 implies that there is $l \in \mathbb{N}$ and a recurrent vertex $w$ such that $(w, x) \in R^{l}$. Hence the definitions of $E$ and $\geq$ imply $[w] \geq[v]$, where $[v]$ denotes the equivalent class (w.r.t. $E$ ) containing the vertex $v$. Since $[v]$ is maximal w.r.t. $\geq$, we have $[v]=[w]$. Consequently, there exists an index $k \in \mathbb{N}$ such that $(v, w) \in R^{k}$. On the other hand, we have $(x, x)=(x, v) \circ(v, w) \circ(w, x) \in R^{k+l+1}$. Thus, $x$ is recurrent, a contradiction.

Therefore, every vertex $x \in R^{-1}(v)$ is recurrent. Hence $[x] \geq[v]$ together with the maximality of $[v]$ gives $[x]=[v]$, and thus $x \in V_{G_{r}}$.

Lemma 4.6. For every $x \in V_{G}$, there is $l \in \mathbb{N}$ such that, for arbitrary $i \geq l$, there exists $u \in V_{G_{r}}$ satisfying $(u, x) \in R^{i}$.

Proof. From Lemma 4.4 and Lemma 4.5 we conclude that it is sufficient to show that for an arbitrary recurrent vertex $v$ there is a $k \in \mathbb{N}$ and $w \in V_{G_{r}}$ such that $(w, v) \in R^{k}$. The lemma now follows easily from the finiteness of $V_{G}$.

From these three lemmata we can deduce

Theorem 4.7. All solutions of $G * R=G$ are automorphisms if and only if $G$ has property $N$. 
Proof. Suppose there are distinct vertices $x, y \in V_{G}$ such that $N_{G}(x) \subseteq N_{G}(y)$. Then $R=I_{G} \cup(x, y)$, which is not functional, satisfies $G * R=G$. Thus $G * R=G$ is also solved by relations that are not automorphisms of $G$. This proves the "only if" part.

Conversely, suppose $G$ has property N. Claim: There is a $k \in \mathbb{N}$ such that $R^{k} \cap\left(V_{G_{r}} \times\right.$ $\left.V_{G_{r}}\right)=I_{G_{r}}$.

For each $v_{i} \in V_{G_{r}}$ there is a walk of length $s_{i} \geq 1$ from $v_{i}$ to itself. Hence $\left(v_{i}, v_{i}\right) \in$ $R^{s_{i}}$. Let $s$ be the least common multiple of the $s_{i}$. Then $\left(v_{i}, v_{i}\right) \in R^{s}$ for all $v_{i} \in V_{G_{r}}$. Define $Q:=R^{s} \cap\left(V_{G_{r}} \times V_{G_{r}}\right)$. Thus $I_{G_{r}} \subseteq Q$ and moreover $Q^{j} \subseteq Q^{j+1}$ for all $j \in \mathbb{N}$. Since $V_{G_{r}}$ is finite there is an $n \in \mathbb{N}$ such that $Q^{n+1}=Q^{n}$, and hence $Q^{2 n}=Q^{n}$. Let us write $R^{-i}(v):=\left\{u \in V_{G}:(u, v) \in R^{i}\right\}$. For $v \in V_{G_{r}}$ we have $R^{-i}(v) \in V_{G_{r}}$ (from Lemma 4.5) and hence $Q^{-n}(v)=R^{-s n}(v)$ for all $v \in V_{G_{r}}$. If $Q^{n} \neq I_{G_{r}}$, then there are two distinct vertices $u, v \in V_{G_{r}}$, such that $(u, v) \in Q^{n} . N_{G}(u) \nsubseteq N_{G}(v)$ and $G=G * R^{s n}$ allows us to conclude that $R^{-s n}(u) \nsubseteq R^{-s n}(v)$ and $R^{-s n}(v) \nsubseteq R^{-s n}(u)$. Hence, there is a vertex $w$, such that $(w, u) \in Q^{n}$ and $(w, v) \notin Q^{n}$. From $(u, v) \in Q^{n}$ and $(w, u) \in Q^{n}$ we conclude $(w, v) \in Q^{n} \circ Q^{n}=Q^{2 n}$, contradicting to $Q^{2 n}=Q^{n}$. Therefore $Q^{n}=I_{G_{r}}$. Setting $k=s n$ now implies the claim.

Finally, we show $V_{G_{r}}=V_{G}$. For any $v \in V_{G} \backslash V_{G_{r}}$, Lemma 4.6 implies the existence of $w \in V_{G_{r}}$ and $m \in \mathbb{N}$ such that $(w, v) \in R^{m k}$. However, we have claimed $R^{-k}(w)=\{w\}$, hence $R^{-m k}(w)=\{w\}$. This, however, implies $N_{G}(w) \subseteq N_{G}(v)$ and thus contradicts property $\mathrm{N}$. Therefore, $V_{G}=V_{G_{r}}$ and moreover $R^{k}=I_{G}$. This $R$ is an automorphism.

\section{R-retraction}

A particularly important special case of ordinary graph homomorphisms are homomorphisms to subgraphs, and in particular so-called retractions: Let $H$ be a subgraph of $G$, a retraction of $G$ to $H$ is a homomorphism $r: V_{G} \rightarrow V_{H}$ such that $r(x)=x$ for all $x \in V_{H}$.

We introduced the graph cores in section 3 as minimal representatives of the homomorphism equivalence classes. The classical and equivalent definition is the following: A (graph) core is a graph that does not retract to a proper subgraph. Every graph $G$ has a unique core $H$ (up to isomorphism), hence one can speak of $H$ as the core of $G$, see [7].

Here, we introduce a similar concept based on relations between graphs. Again to obtain a structure related to graph homomorphisms, in this section we require all relations to have full domain unless explicitly stated otherwise.

Definition 5.1. Let $H$ be a subgraph of $G$. An $R$-retraction of $G$ to $H$ is a relation $R$ such that $G * R=H$ and $(x, x) \in R$ for all $x \in V_{H}$. If there is an R-retraction of $G$ to $H$ we say that $H$ is a retract of $G$.

Lemma 5.2. If $H$ is an R-retract of $G$ and $K$ is an R-retract of $H$, then $K$ is an R-retract of $G$.

Proof. Suppose $T$ is an R-retraction of $H$ to $K$ and $S$ is an R-retraction of $G$ to $H$. Then $(G * S) * T=G *(S \circ T)=K$. Furthermore $(x, x) \in T$ for all $x \in V_{K} \subseteq V_{H}$, and $(u, u) \in S$ for all $u \in V_{H}$, hence $(x, x) \in S \circ T$ for all $x \in V_{k}$. Therefore $S \circ T$ is an R-retraction from $G$ to $K$.

Hence, the following definition is meaningful. 
Definition 5.3. A graph is $R$-reduced if there is no R-retraction to a proper subgraph.

Thus, we can also speak about "the R-reduced graph of a graph $G$ " as the smallest subgraph on which it can be retracted. We shall see below that the R-reduced graph of a graph is always unique up to isomorphism.

We shall remark that R-reduced graphs differs from R-cores introduced in section 3, thus we choose an alternative name used also in homomorphism setting (cores are also called reduced graphs).

Lemma 5.4. Let $G$ be a graph with loops and $o$ a vertex of $G$ with a loop on it. Then the R-reduced graph of $G$ is the subgraph induced by $\{o\}$.

Proof. Let $O$ be the graph induced by $\{o\}$, and $R=\left\{(x, o) \mid x \in V_{G}\right\}$, then it is easily seen $R$ is a R-retraction of $G$ to $O$. Moreover, since $O$ has only one vertex, thus there is no R-retraction to its subgraphs. So $O$ is a R-reduced graph of $G$.

Conversely, let $H$ be a R-reduced graph of $G$ and denote by $R$ the R-retraction from $G$ to $H$. Then a loop of $G$ must generate a loop of $H$ via $R$, denote it by $O$. Similarly to above, we see $O$ is a R-retract of $H$, hence it is also a R-retract of $G$ (by Lemma 5.2). Therefore the definition of R-reduced graph implies $H=O$.

In the remainder of this section, therefore, we will only consider graphs without loops.

Lemma 5.5. If $G$ is R-reduced, then $G$ has property N.

Proof. Suppose there are two distinct vertices $x, y \in V_{G}$ with $N_{G}(x) \subseteq N_{G}(y)$ and consider the induced graph $G / x:=G\left[V_{G} \backslash\{x\}\right]$ obtained from $G$ by deleting the vertex $x$ and all edges incident with $x$. The relation $R=\left\{(z, z) \mid z \in V_{G} \backslash\{x\}\right\} \cup\{(x, y)\}$ satisfies $G * R=G / x$ : the first part is the identity on $G / x$ and already generates all necessary edges in $G / x$. The second part transforms edges of the form $(x, z) \in E_{G}$ to edges $(y, z)$. Since $R$ has full domain and contains the identity relation restricted to $G / x$, it is an R-retraction of graph $G$, and hence $G$ is not R-reduced.

Proposition 5.6. A graph $G$ is R-reduced if and only if it has no relation to a proper subgraph.

Proof. The "if" part is trivial. Now we suppose that $H$ is a proper induced subgraph of graph $G$ with the minimal number of vertices such that there is a relation $R$ satisfying $G * R=H$. Then $H$ does not have a relation to a proper subgraph of itself. We claim that $H$ has property $\mathrm{N}$; otherwise, one can find a vertex $u \in V_{H}$ and construct a retraction from $H$ to $H / u$ as in Lemma 5.5, which causes a contradiction. Denote $\tilde{R}=R \cap\left(V_{H} \times V_{H}\right)$, then $K=H * \tilde{R}$ is a subgraph of $H$. From our assumptions on $H$ we obtain $K=H$. By virtue of Theorem 4.7, $\tilde{R}$ is induced by an automorphism of $H$. Hence $R \circ \tilde{R}^{+}$is again a relation of $G$ to $H$ that contains the identity on $H$, i.e., it is an R-retraction.

Since graph cores are induced subgraphs and retractions are surjective they also imply relations. Proposition 5.6 is also a consequence of this fact. We refer to [7] for a formal proof.

We call $R$ a minimal $R$-retraction if there is no R-retraction $R^{\prime}$ such that $R \supset R^{\prime} \supset I_{H}$.

Lemma 5.7. Let $H$ be an R-retract of $G$. Then any minimal R-retraction of $G$ to $H$ is functional. 

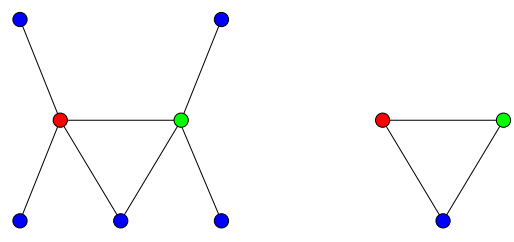

Figure 5: A graph $G$ and its core.

Proof. Suppose $R$ is a minimal R-retraction of $G$ to $H$. If $R$ is not functional, then there exist distinct $x, y \in V_{H}$ such that $(u, x),(u, y) \in R$. Hence we could always pick a vertex from $\{x, y\}$ which is different of $u$, w.l.o.g. suppose it is $x$. Then $R /(u, x)$ is an R-retraction, which contradicts minimality. To see this, set $R^{\prime}=R /(u, x)$, then $R \supset R^{\prime} \supset$ $I_{H}$ and moreover $H=G * I_{H} \subseteq G * R^{\prime} \subseteq G * R=H$, and thus $G * R^{\prime}=H$.

Proposition 5.8. A graph is R-reduced if and only if it is a graph core.

Proof. If $H$ is R-reduced from $G$ there is an R-retraction from $G$ to $H$ which can be chosen minimal and hence by Lemma 5.7 is functional and hence is a homomorphism retraction. Conversely, a homomorphism retraction is also an R-retraction. Hence the Rreduced graphs coincide with the graph cores.

Proposition 5.9. Suppose $H$ is the core of graph $G$. If $H * R=K$ then there is a relation $R^{\prime}$ such that $G * R^{\prime}=K$. If $K * S=G$, then there is a relation $S^{\prime}$ such that $K * S^{\prime}=H$.

Proof. Since $H$ is the core of graph $G$, there is a relation $R_{1}$ such that $G * R_{1}=H$. If $H * R=K$ we have $G * R_{1} * R=K$ and $R^{\prime}=R_{1} \circ R$ satisfies $G * R^{\prime}=K$. If $K * S=G$ we have $K * S * R_{1}=H$ and $S^{\prime}=S \circ R_{1}$ satisfies $K \circ S^{\prime}=G$.

\subsection{Cocores}

In the classical setting of maps between graphs, one can only consider retractions from a graph to its subgraphs, since graph homomorphisms of an induced subgraph to the original graph are just the identity maps. In the setting of relations between graphs, however, it appears natural to consider relations with identity restriction between a graph and an induced subgraph. This gives rise to notions of R-coretraction and R-cocore in analogy with R-retractions and R-reduced graphs.

Definition 5.10. Let $H$ be a subgraph of graph $G$. An $R$-coretraction of $H$ to $G$ is a relation $R$ such that $H * R=G$ and $(x, x) \in R$ for all $x \in V_{H}$. We say that $H$ is an $R$-coretract of $G$.

Lemma 5.11. If $H$ is an R-coretract of graph $G$ and $K$ is an R-coretract of $H$, then $K$ is an R-coretract of $G$.

Proof. Suppose $T$ is an R-coretraction of $K$ to $H$ and $S$ is an R-coretraction of $H$ to $G$. Then $(K * T) * S=K *(T \circ S)=G$. Furthermore $(x, x) \in T$ for all $x \in V_{K} \subseteq V_{H}$, and $(v, v) \in S$ for all $v \in V_{H}$, hence $(x, x) \in T \circ S$ for all $x \in V_{K}$. Therefore $T \circ S$ is an R-coretraction from $K$ to $G$. 
Hence, the following definition is meaningful.

Definition 5.12. An R-coretract $H$ of a graph $G$ is an $R$-cocore of $G$ if $H$ does not have a proper subgraph that is an R-coretract of $H$ (and hence of $G$ ).
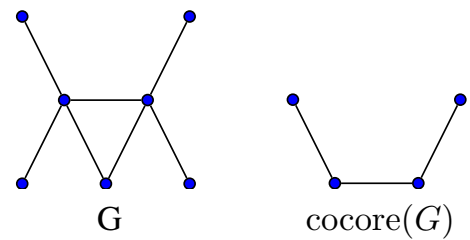

Figure 6: A graph and its cocore

Clearly, the reference to $G$ is irrelevant: A graph $G$ is an $R$-cocore if there is no proper subgraph of $G$ that is an R-coretract of $G$. Similarly, we call $R$ to be a minimal $R$-coretraction of $H$ to $G$ if there exists no R-coretraction $R^{\prime}$, such that $R^{\prime} \subset R$.

Lemma 5.13. Let $H$ be an R-coretract of graph $G$, and let $R$ be a minimal R-coretraction of $H$ to $G$. Then the restriction of $R$ to $H$ equals $I_{H}$.

Proof. Suppose $R \cap\left(V_{H} \times V_{H}\right) \neq I_{H}$ and define $R_{1}=R \backslash\left\{(x, y) \in R: x, y \in V_{H}, x \neq\right.$ $y$ \}. Then $H * R_{1} \subseteq H * R=G$. We claim that $H * R_{1}=H * R$ and thus $R_{1}$ is an R-coretraction of $H$ to $R$, contradicting the minimality of $R$.

To prove this claim, it is sufficient to show that any edge $e \in E_{G}$ is contained in $H * R_{1}$. If $e$ is not incident with any vertex in $V_{H}$ or $e \in E_{H}$, the conclusion is trivial. So we only need to consider $e=(z, u)$ with $z \in E_{H}$ and $u \in V_{G} \backslash V_{H}$. Since $G=H * R$, one can find $x_{1}, x_{2} \in V_{H}$ such that $\left(x_{1}, z\right),\left(x_{2}, u\right) \in R$ and $\left(x_{1}, x_{2}\right) \in E_{H}$. Because $H \subseteq H *\left(I_{H} \cup\left(x_{1}, z\right)\right) \subset H *\left(R \cap\left(V_{H} \times V_{H}\right)\right)=H$, we get $N_{H}\left(x_{1}\right) \subseteq N_{H}(z)$. It follows that $\left(z, x_{2}\right) \in E_{H}$ and hence $e=(z, u) \in G * R_{1}$.

Like R-reduced graphs, R-cocores satisfy a stringent condition on their neighborhood structure.

Definition 5.14. A graph $G$ satisfies property $\mathrm{N}^{*}$ if, for every vertex $x \in V_{G}$, there is no subset $U_{x} \subseteq V_{G} \backslash\{x\}$ such that

$$
N_{G}(x)=\bigcup_{y \in U_{x}} N_{G}(y)
$$

In other words, no neighborhood can be represented as the union of neighborhoods of other vertices of graph $G$.

Proposition 5.15. $G$ is an R-cocore if and only if $G$ has property N*.

Proof. Consider a vertex set $U_{x}$ as in Definition 5.14 and suppose that there is a vertex $x \in V_{G}$ such that $N_{G}(x)=\bigcup_{y \in U_{x}} N_{G}(y)$. Then the relation $R:=I \backslash(x, x) \cup\{(y, x)$ : $\left.y \in U_{x}\right\}$ is an R-coretraction from $G / x$ to $G$. Thus $G$ is not a R-cocore.

Conversely, suppose that $G$ is not an R-cocore, let $H$ be a coretract of $G$, and denote by $R$ a minimal R-coretraction of $H$ to $G$. Then, by Lemma 5.13, $R \cap\left(V_{H} \times V_{H}\right)=I_{H}$. Consider a vertex $v \in V_{G} \backslash V_{H}$ and set $R^{-1}(v)=\left\{x_{1}, \cdots, x_{i}\right\}$. Then $N(v)=\bigcup_{i} N\left(x_{i}\right)$, contradicting property $\mathrm{N}^{*}$. 
Proposition 5.16. The R-cocore of $G$ is unique up to isomorphism.

Proof. We denote by $\mathcal{N}$ the collection of all open neighborhoods of vertices in $G$, i.e., $\mathcal{N}=\left\{N_{G}\left(x_{1}\right), N_{G}\left(x_{2}\right), \cdots, N_{G}\left(x_{k}\right)\right\}$, where $V_{G}=\left\{x_{1}, x_{2}, \cdots, x_{k}\right\}$. From the definition of the R-cocore we know that the subcollection $\mathcal{M}$ of $\mathcal{N}$ consisting of all the open neighborhoods of vertices in R-cocore is a basis of $\mathcal{N}$, i.e., any set in $\mathcal{N}$ can be expressed by the union of some sets in $\mathcal{M}$. W.l.o.g., we denote the vertex set in a R-cocore $C$ of $G$ is $\left\{x_{1}, x_{2}, \cdots, x_{m}\right\}$ where $m \leq k$, then $\mathcal{M}=\left\{N_{G}\left(x_{1}\right), N_{G}\left(x_{2}\right), \cdots, N_{G}\left(x_{m}\right)\right\}$. We claim that any element in $\left\{N_{G}\left(x_{1}\right), N_{G}\left(x_{2}\right), \cdots, N_{G}\left(x_{m}\right)\right\}$ cannot be expressed as the union of other elements, i.e., $\mathcal{M}$ is a minimal basis. Otherwise, w.l.o.g., suppose $N_{G}\left(x_{1}\right)=\cup_{x_{k}} N_{G}\left(x_{k}\right), x_{k} \in\left\{x_{2}, \ldots, x_{m}\right\}$. For any $1 \leq k \leq m, N_{G}\left(x_{k}\right)=N_{C}\left(x_{k}\right)$ or $N_{G}\left(x_{k}\right)=N_{C}\left(x_{k}\right) \cup\left\{x_{i} \mid\left(x_{i}, x_{k}\right) \in E_{G}, m+1 \leq i \leq n\right\}$, so either $N_{C}\left(x_{1}\right)=$ $\cup_{x_{k}} N_{C}\left(x_{k}\right), x_{k} \in\left\{x_{2}, \ldots, x_{m}\right\}$ or $N_{C}\left(x_{1}\right)=\cup_{x_{k}} N_{C}\left(x_{k}\right) \cup\left\{x_{i} \mid\left(x_{i}, x_{k}\right) \in E_{G}, m+1 \leq\right.$ $\left.i \leq n, x_{k} \in\left\{x_{2}, \ldots, x_{m}\right\}\right\}$, the former contradicts to Proposition 5.15, which implies any element in $\left\{N_{C}\left(x_{1}\right), N_{C}\left(x_{2}\right), \cdots, N_{C}\left(x_{m}\right)\right\}$ cannot be expressed as the union of other elements, the latter is impossible because $\left\{x_{i} \mid\left(x_{i}, x_{k}\right) \in E_{G}, m+1 \leq i \leq n, x_{k} \in\right.$ $\left.\left\{x_{2}, \ldots, x_{m}\right\}\right\} \nsubseteq C$.

Now we prove that this minimal basis is unique. Note that in $\mathcal{N}$ we view any vertex with the same neighborhood as the same, since any vertex in R-cocore has different neighborhoods. Let us consider two minimal sub-collections $\mathcal{A}, \mathcal{B}$. Neither contains the other by their minimality. Since everything is finite, let $A \in \mathcal{A} / \mathcal{B}$ be an element of minimal size. Now $A$ can be expressed as a union of elements of $\mathcal{B}$, which all need to be of smaller cardinality than $A$ (or same but $A \notin \mathcal{B}$ ), but $\mathcal{A}$ then contains all of them, letting $A$ be expressed by a union of elements of $\mathcal{A}$ contradicting the minimality of $\mathcal{A}$.

These results allow us to construct an algorithm that computes the cocore of given graph $G$ in polynomial time. First observe that the cocore of a graph $G$ that contains isolated vertices is the disjoint union of cocore of the graph $G^{\prime}$ obtained from $G$ by removing isolated vertices and the graph consisting of a single isolated vertex. It is thus sufficient to compute cocores for graphs without isolated vertices in Algorithm 2.

Proposition 5.17. Suppose $H$ is a cocore of $G$. If $K * R=H$, then there is a relation $R^{\prime}$ such that $K * R^{\prime}=G$. If $G * S=K$, then there is a relation $S^{\prime}$ such that $H * S^{\prime}=K$.

Proof. Since $H$ is a cocore of $G$, there exists an R-coretraction $R_{1}$ such that $H * R_{1}=G$. If $K * R=H$, then letting $R^{\prime}=R \circ R_{1}$ implies $K * R^{\prime}=G$. If $G * S=K$, we have $H * R_{1} * S=K$. Let $S^{\prime}=R_{1} \circ S$, then $H * S^{\prime}=K$.

\section{Computational complexity}

In this section we briefly consider the complexity of computational problems related to graph homomorphisms. The homomorphism problem $\operatorname{HoM}(H)$ takes as input some finite $G$ and asks whether there is a homomorphism from $G$ to $H$. The computational complexity of the homomorphism problem is fully characterized. It is known that $\operatorname{HoM}(H)$ is NPcomplete if and only if $H$ has no loops and contains odd cycles. All the other cases are polynomial, see [7].

The analogous problem for relations between graphs can be phrased as follows: The full relation problem $\operatorname{FuL-REL}(H)$ takes as input some finite $G$ and asks whether there is a relation with full domain from $G$ and asks whether there is a relation from $G$ to $H$. We 


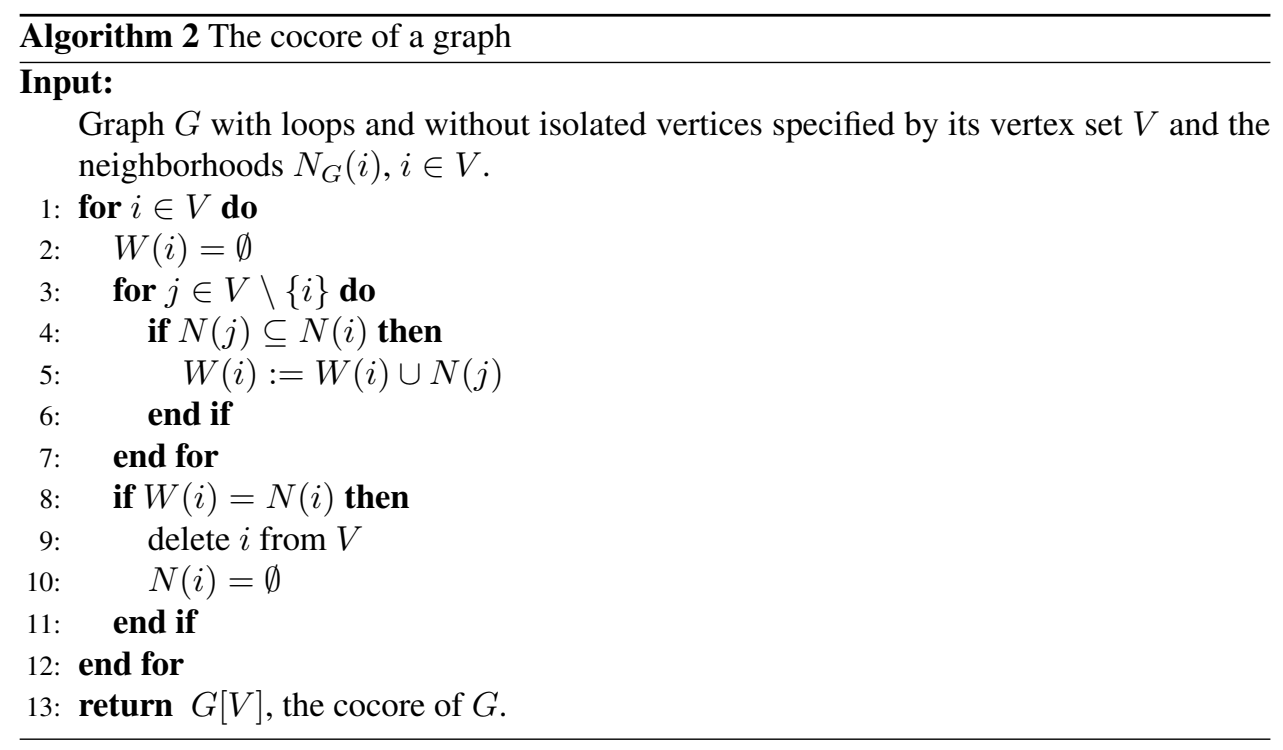

show that this problem can be easily converted to a related problem on surjective homomorphisms. The surjective homomorphism problem $\operatorname{SuR-HOM}(H)$ takes as input some finite $G$ and asks whether there is a surjective homomorphism from $G$ to $H$.

Let $\leq_{\mathrm{P}}^{\text {Tur }}$ indicate polynomial time Turing reduction.

Theorem 6.1. For finite $H$ our relation problem sits in the following relationship.

$$
\operatorname{Hom}(H) \leq_{P}^{\text {Tur }} \operatorname{Ful-REL}(H) \leq_{P}^{\text {Tur }} \operatorname{SuR-HOM}(H) .
$$

Proof. First we show that $\operatorname{Hom}(H)$ is polynomially reducible to $\operatorname{FuL}-\operatorname{ReL}(H)$. If there is a homomorphism from $G$ to $H$, then there is also a surjective homomorphism from $G+H$ to $H$. On the other hand, suppose $G$ has no homomorphism to $H$. From Lemma 1.4 we conclude that $G+H$ has no relation to $H$ since $G$ has no relation to $H$.

The relation problem FUL- $\operatorname{REL}(H)$ is polynomially reducible to $\operatorname{SUR}-\operatorname{Hom}(H)$. From Corollary 2.3 we know $G * R=H$ if and only if there is a graph $G^{\prime}=G * R_{D}$ which has a full homomorphism to $G$ and has a surjective homomorphism to $H$.

We construct $G^{\prime \prime}$, by duplicating all the vertices of $G$ precisely $\left|V_{H}\right|$ times. It is easy to see that if $G^{\prime}$ exists, we can also put $G^{\prime}=G^{\prime \prime}$ because the surjective homomorphism can easily undo the redundant duplications.

It remains to check whether there is surjective homomorphism from $G^{\prime \prime}$ to $H$. This gives the polynomial reduction from $\operatorname{FuL}-\operatorname{REL}(H)$ to $\operatorname{SuR}-\operatorname{Hom}(H)$.

To our knowledge, SuR-Hom $(H)$ is not fully classified. A recent survey of the closely related complexity problem concerning the existence of vertex surjective homomorphisms [2] provides some arguments why the characterization of complexity is likely to be hard, see also [5]. We observe that the existence of a homomorphism from $G$ to $H$ is equivalent to the existence of a surjective homomorphism from $G+H$ to $H$. Thus $\operatorname{SuR-Hom}(H)$ is clearly hard for all graphs for which $\operatorname{Hom}(H)$ is hard, i.e., for all loop-less graphs with odd cycles. 
Testing the existence of a homomorphism from a fixed $G$ to $H$ is polynomial (there is only a polynomial number $\left|V_{H}\right|^{\left|V_{G}\right|}$ of possible functions from $G$ to $H$ ). Similarly the existence of a relation from a fixed $G$ to $H$ is also polynomial. In fact, an effective algorithm exists. For fixed $G$ there are finitely many thin graphs $T$ which $G$ has relation to. The algorithm thus first constructs the thin graph of $H$ and then, using a decision tree recognizes all isomorphic copies of all thin graphs $G$ has relation to.

\section{Weak relational composition}

In this section we will briefly discuss the "loop-free" version, i.e., equations of the form $G \star R=H$.

Most importantly, there is no simple composition law analogous to Lemma 2.1. The expression

$$
(G \star R) \star S=\left(S^{+} \circ\left(R^{+} \circ G \circ R\right)^{\iota} \circ S\right)^{\iota}
$$

does not reduce to relational composition in general. For example, let $G=K_{3}$ with vertex set $V=\{x, y, z\}$ and consider the relations $R=\{(x, 1),(z, 1),(y, 2)\} \subseteq\{x, y, z\} \times$ $\{1,2\}$ and $S=\left\{\left(1, x^{\prime}\right)\left(1, z^{\prime}\right)\left(2, y^{\prime}\right)\right\} \subseteq\{1,2\} \times\left\{x^{\prime}, y^{\prime}, z^{\prime}\right\}$. One can easily verify

$$
(G \star R) \star S=P_{2} \neq G \star(R \circ S)=K_{3}
$$

The most important consequence of the lack of a composition law is that R-retractions cannot be meaningfully defined for the weak composition. Similarly, the results related to R-equivalence heavily rely on the composition law.

Nevertheless, many of the results, in particular basis properties derived in section 2, remain valid for the weak composition operation. As the proofs are in many cases analogous, we focus here mostly on those results where strong and weak composition differ, or where we need different proofs. In particular, Lemma 2.2 also holds for the weak composition. Thus, we still have a result similar to corollary 2.3 , but the proof is slightly different.

Corollary 7.1. Suppose $G \star R=H$. Then there is a set $C$, an injective relation $R_{D} \subseteq$ $\operatorname{dom} R \times C$, and a surjective relation $R_{C} \subseteq C \times \operatorname{img} R$ such that $G[\operatorname{dom} R] \star R_{D} \star R_{C}=$ $H[\operatorname{img} R]$.

Proof. From Proposition 2.2 we know $R=I^{\prime} \circ R_{D} \circ R_{C} \circ I^{\prime \prime}$. And we know $G[\operatorname{dom} R] \star$ $R_{D}=G[\operatorname{dom} R] * R_{D}$. From the properties of $\star$, we have

$$
\begin{aligned}
G[\operatorname{dom} R] \star R & =\left(R^{+} \circ G[\operatorname{dom} R] \circ R\right)^{l} \\
& =\left(\left(R_{D} \circ R_{C}\right)^{+} \circ G[\operatorname{dom} R] \circ R_{D} \circ R_{C}\right)^{l} \\
& =\left(R_{C}^{+} \circ R_{D}^{+} \circ G[\operatorname{dom} R] \circ R_{D} \circ R_{C}\right)^{l} \\
& =\left(R_{C}^{+} \circ\left(R_{D}^{+} \circ G[\operatorname{dom} R] \circ R_{D}\right) \circ R_{C}\right)^{l} \\
& =\left(R_{C}^{+} \circ G[\operatorname{dom} R] * R_{D} \circ R_{C}\right)^{l} \\
& =\left(R_{C}^{+} \circ G[\operatorname{dom} R] \star R_{D} \circ R_{C}\right)^{l} \\
& =G[\operatorname{dom} R] \star R_{D} \star R_{C} \\
& =H[\operatorname{img} R]
\end{aligned}
$$


Assume $G \star R=H$ and let $H_{1}, \cdots, H_{k}$ the connected components of $H$. From the definition of $\star$ and $*$, if we denote $\tilde{H}=G * R$, then $\tilde{H}$ could be decomposed into the union of connected components $\tilde{H}_{i}(1 \leq i \leq k)$, such that $\left(\tilde{H}_{i}\right)^{\iota}=H_{i}$. Hence the conclusion of the proposition 2.4 also holds true for weak relations.

Lemma 2.6 does not hold for weak relations. For example, there is a weak relation of $K_{5}$ to $K_{3}$, but $\chi\left(K_{5}\right)=5>\chi\left(K_{3}\right)=3$.

Lemma 2.7 and Lemma 2.8 do not hold for weak relations. For example, if $G$ is a graph consisting of a single isolated vertex, then $P_{3} \star R=G$ and $C_{3} \star R=G$, but there are no walk in $G$.

With respect to complete graphs, weak relational composition also behaves different from strong composition. If $K_{k} \star R=H$ then $R(i)$ can contain more that one vertex in $V_{H}$. Compared to Proposition 2.12, we also obtain a different result:

Theorem 7.2. There is a relation $R$ such that $K_{k} \star R=H$ if and only if every connected component of $\bar{H}$ is a complete graph, and the number of connected components of $\bar{H}$ containing at least 2 vertices is at most $k$.

Proof. If every connected component of $\bar{H}$ is a complete graph, denoted the vertex sets of the connected components containing at least 2 vertices by $H_{1}, \ldots, H_{m}, m \leq k$ and the vertices of $K_{k}$ by $1, \cdots, k$. Let $R=\left\{(i, u) \mid i=1, \cdots, k, u \in V_{H_{i}}\right\} \cup\{(j, v): 1 \leq j \leq$ $\left.k, v \in V_{H} \backslash \bigcup_{i=1}^{m} V_{H_{i}}\right\}$. One easily checks that $K_{k} \star R=H$.

Conversely, let $R$ be a relation satisfying $K_{k} \star R=H$. Consider the set $U_{i}=\{u \in$ $\left.V_{H} \mid R^{-1}(u)=\{i\}\right\}$. Then $u$ and $v$ are not adjacent for arbitrary $u, v \in U_{i}$, while $u$ is adjacent to $w$ for every $w \in V_{H} \backslash U_{i}$. Hence $\bar{H}\left(U_{i}\right)$ is a connected component of $\bar{H}$, which is also a complete graph. Given $w \in V_{H} \backslash \bigcup_{i=1}^{m} U_{i}, R^{-1}(w)$ must have at least 2 vertices in $K_{k}$, hence $w$ is adjacent to every vertex in $H$ except itself; in other words, $w$ is an isolated vertex in $\bar{H}$. Therefore the number of connected components of $\bar{H}$ containing at least 2 vertices is no more than $k$.

The results in subsection 3.1 also remain true for weak relations.

\section{Acknowledgments}

We thank Rostislav Matveev for helpful discussions in the beginning of the project and pointing out the decomposition as in Lemma 2.3, and to Jaroslav Nešetřil for pointing out the equivalence of some complexity problems and enlightening questions for further works. L.Y. is grateful to the Max Planck Institute for Mathematics in the Sciences in Leipzig for its hospitality and continuous support. This work was supported in part by the NSFC (to L.Y.), the VW Foundation (to J.J. and P.F.S.), the Czech Ministry of Education, and ERCCZ LL-1201, and CE-ITI of GAČR (to J.H).

\section{References}

[1] E. Babson and D. Kozlov, Complexes of graph homomorphisms, Israel J. Math. 152 (2006), 285-312.

[2] M. Bodirsky, J. Kára and B. Martin: The complexity of surjective homomorphism problems a survey, Discr. Appl. Math. 160 (2012), 1680-1690.

[3] S. N. Dorogovtsev and J. F. F. Mendes, Evolution of Networks: From Biological Nets to the Internet and $W W W$, Oxford Univ. Press, Oxford, UK, 2003. 
[4] T. Feder and P. Hell, On realizations of point determining graphs, and obstructions to full homomorphisms, Discrete Math. 308 (2008), 1639-1652.

[5] P. A. Golovach, B. Lidický, B. Martin and D. Paulusma: Finding vertex-surjective graph homomorphisms, Acta Informatics 49 (2012), 381-394.

[6] R. Hammack, W. Imrich and S. Klavžar, Handbook of Product Graphs, Discrete Mathematics and Its Applications, CRC Press, Boca Raton, FL, 2011.

[7] P. Hell and J. Nešetřil, Graphs and homomorphisms, Oxford University Press, Oxford, UK, 2004.

[8] J. Hubička, J. Fiala and Y. Long: Constrained homomorphism orders, in preparation, 2012.

[9] W. Imrich and S. Klavžar, Product Graphs: Structure and Recognition, Wiley, New York, 2000.

[10] J. Matoušek, Using the Borsuk-Ulam Theorem, Springer, Berlin, DE, 2003.

[11] M. E. J. Newman, Networks: An Introduction, Oxford Univ. Press, Oxford, UK, 2010.

[12] A. Schrijver, Combinatorial optimization: B, Springer, Berlin, 2003.

[13] D. P. Sumner, Point determination in graphs, Discrete Mathematics 5 (1973), 179-187.

[14] S. Wuchty and E. Almaas, Evolutionary cores of domain co-occurrence networks, BMC Evol. Biol. 5 (2005), 24.

[15] Y. Xiao, B. D. MacArthur, H. Wang, M. Xiong and W. Wang, Network quotients: Structural skeletons of complex systems, Phys. Rev. E 78 (2008), 046102.

[16] A. Zhang: Protein interaction networks: computational analysis. Cambridge University Press, Cambridge, UK, 2009. 